\title{
LHC Collider Phenomenology of Minimal Universal Extra Dimensions
}

\author{
Jyotiranjan Beuria, ${ }^{a, b, c}$ AseshKrishna Datta, ${ }^{a, b}$ Dipsikha Debnath, ${ }^{d}$ Konstantin \\ T. Matchev ${ }^{d}$ \\ ${ }^{a}$ Harish-Chandra Research Institute, Allahabad 211019, India \\ ${ }^{b}$ Homi Bhabha National Institute, Training School Complex, Anushakti Nagar, \\ Mumbai 400085, India \\ ${ }^{c}$ Regional Centre for Accelerator-based Particle Physics, Harish-Chandra Research Institute, \\ Allahabad 211019, India \\ ${ }^{d}$ Physics Department, University of Florida, Gainesville, FL 32611, USA
}

\begin{abstract}
We discuss the collider phenomenology of the model of Minimal Universal Extra Dimensions (MUED) at the Large hadron Collider (LHC). We derive analytical results for all relevant strong pair-production processes of two level 1 Kaluza-Klein partners and use them to validate and correct the existing MUED implementation in the fortran version of the Pythia event generator. We also develop a new implementation of the model in the $\mathrm{C}++$ version of Pythia. We use our implementations in conjunction with the Checkmate package to derive the LHC bounds on MUED from a large number of published experimental analyses from Run 1 at the LHC.
\end{abstract}




\section{Contents}

1 Introduction 1

2 MUED preliminaries $\quad 6$

2.1 Notations and conventions $\quad 6$

2.2 Mass spectrum uncertainties $\quad 6$

3 Strong production in MUED $\quad 8$

3.1 Analytical results in the limit of degenerate level $1 \mathrm{KK}$ partners 9

$\begin{array}{lll}3.2 & \text { Pythia6 validation } & 10\end{array}$

$\begin{array}{lll}3.3 & \text { The relative importance of virtual level } 2 \text { KK particles } & 14\end{array}$

4 Constraints on MUED $\quad 14$

$\begin{array}{lll}4.1 & \text { Constraints from cosmology } & 14\end{array}$

$\begin{array}{lll}4.2 & \text { LHC simulation details } & 16\end{array}$

$\begin{array}{lll}4.3 & \text { LHC bounds } & 18\end{array}$

$\begin{array}{llr}5 & \text { Conclusions } & 19\end{array}$

A KK gluon and KK quark production: the mass-nondegenerate case 20

B Implementation of MUED in Pyтніа6 23

$\begin{array}{ll}\text { C Implementation of MUED in PYTHiA8 } & 25\end{array}$

\section{Introduction}

The ongoing run of the Large Hadron Collider (LHC) at CERN is continuing its quest for new physics Beyond the Standard Model (BSM). From a theorist's viewpoint, the first step in this program is to characterize the anticipated new physics in terms of its particle content, interactions, and parameter values at the relevant energy scale of the collider experiment [1]. ${ }^{1}$ Traditionally, this can be done in one of two ways: by considering a "complete" model, or a "simplified" model.

- Complete models. Here one introduces the complete new physics structure which exists below the ultraviolet cutoff of the effective theory. The standard and most popular example of this type is the framework of supersymmetry (SUSY) [4]. Unfortunately, this

\footnotetext{
${ }^{1}$ Two alternative model-independent approaches, where one does not have to specify a particular BSM model, have recently been proposed in Refs. [2, 3].
} 
approach usually brings about a large number of input parameters; for example, even the minimal version of SUSY, the Minimal Supersymmetric Standard Model (MSSM), has more than 100 input parameters [5], which need to be trimmed down to a more manageable number. This can be done in two ways:

1. Phenomenological models. Here, one retains full generality, but fixes some subset of the original input parameters to some typical values which are motivated or dictated by the current experimental limits. For example, the general MSSM parameter space is severely constrained by the existing bounds from CP-violating and flavor-changing processes, thus a reasonable and conservative approach is to set the corresponding $\mathrm{CP}$-violating and flavor-changing parameters to zero, achieving a drastic reduction in the dimensionality of the relevant parameter space $[6,7]$. This simplification has allowed the LHC collaborations to publish several analyses from SUSY searches in the thus obtained "phenomenological MSSM" (pMSSM) [8-10].

2. Models with high-scale boundary conditions. Alternatively, one can choose to specify the model parameters at the matching scale, where the ultraviolet completion of the theory is expected to impose certain restrictions on the allowed pattern of parameters. The most celebrated example of this type is a grand unification theory (GUT), which unifies the values for the gauge and certain Yukawa couplings at the GUT scale. In SUSY GUTs, the soft masses for the SUSY partners are also unified according to irreducible representations of the GUT group, as in the minimal supergravity (mSUGRA) model, for example [11-14]. However, this approach also requires a procedure to relate the handful of theory input parameters at the high scale to the phenomenological model parameters at the electroweak scale. The procedure involves numerically solving the renormalization group equations, minimizing the effective potential, and computing the radiative corrections to the particle mass spectrum. In the case of SUSY, several programs have been developed to perform these tasks: ISAJET [15], SOFTSUSY [16], SUSpect [17], and SPheno [18]. Since the calculations are done at a fixed order in perturbation theory, some differences between the programs inevitably arise, due to a different set of higher order terms being neglected. In the past, these differences were carefully scrutinized for the case of the MSSM, which led to an estimate of the inherent theoretical systematic error in these types of calculations in SUSY [19, 20].

- Simplified models. Given that phenomenological models have too many input parameters, while models with high-scale boundary conditions involve unverified theoretical assumptions, the recent trend has been to consider the so-called simplified models, where one focuses only on one specific event topology of interest, and treats the masses of the BSM particles in it (as well as the overall rate) as free parameters [21]. This approach is a compromise between the previous two - on the one hand, the number of input parameters is greatly reduced, since only a few BSM states are present in a given event topology, while at the same time, the mass parameters can be treated in full 
generality, without any a priori theoretical assumptions. This has motivated the LHC collaborations to publish simplified model interpretations of their new physics searches as well [22-25]. The main goal of the simplified model approach is to allow for published LHC results to be easily recast for a variety of BSM scenarios, e.g. with different couplings, spins, etc. However, all these benefits do come at a certain cost - for any given complete model, the limits derived from an exclusive analysis of a single simplified model topology will be very conservative, since each subprocess typically contributes only a small fraction to the rate for the inclusive final state signature being studied. The bounds are therefore generally underestimated and to obtain the true limit in a given complete model, one has to combine the results from several simplified model analyses $[26,27]$, reintroducing the problems mentioned above.

For many years, the only complete models which were seriously investigated by the Tevatron and LHC collaborations, were different versions of low energy supersymmetry: mSUGRA, gauge-mediated SUSY [28], anomaly-mediated SUSY [29, 30], etc. Apart from its attractive features from a theorist's point of view, the ubiquity of supersymmetry was also partially due to the fact that it was readily available in the existing Monte Carlo simulation tools used by the experimental collaborations. However, with the recent progress on automation and standardization ${ }^{2}$ of the simulation chain leading from a theory Lagrangian to fully simulated events [1], this restriction has largely been lifted, and a much wider class of models can now be explored.

Our main objective in this paper is to consider a complete model which is different from supersymmetry, and study its LHC phenomenology. We choose the scenario of Universal Extra Dimensions (UED) [36] in its minimal version, Minimal UED (MUED) [37]. Minimal UED bears some similarities with supersymmetry: for example, each particle of the Standard Model has heavier Kaluza-Klein (KK) partners with the same gauge quantum numbers. Yet there are important differences as well: e.g., the spins of the KK partners differ by $1 / 2$ from those of the superpartners. Unfortunately, this feature is not unique to UED, but is also shared, e.g., by Little Higgs models with $T$-parity [38]. Furthermore, the spin determination of new particles in cascade decays is a notoriously difficult task [39-41], which is expected to take some time after the initial discovery. Thus, given the experimental challenges in discriminating SUSY from UED [42-45], we believe that MUED should be seriously considered as a "complete model" benchmark, on equal footing with SUSY.

At this point in time, studies of MUED are additionally motivated by the null results from the new physics searches at the LHC. In the last couple of years, there has been a flurry of activity in designing models which "hide" the new physics from the LHC. One of the standard methods for doing so is to arrange for a "compressed" mass spectrum with a mass degeneracy of the relevant SUSY particles, so that the resulting decay products are too soft to be triggered upon and tagged in the experimental analysis [46-52], or a "stealth" mass spectrum, where the new physics signature becomes identical to the SM background, since

\footnotetext{
${ }^{2}$ For example, the original SUSY Les Houches accord [31, 32] has now been extended to general BSM models [33-35].
} 
the additional particles are too soft to make any appreciable difference [53-56]. In models with many input parameters like the MSSM, such degeneracies, while phenomenologically desirable, from a theoretical point of view appear accidental and unmotivated. In contrast, at each KK level, the new particle mass spectrum in MUED is very degenerate, and for a good reason - the dominant contribution to the mass comes from the compactification, $m \sim R^{-1}$, where $R$ is the size of the extra dimension. Therefore, MUED represents a well motivated benchmark for a complete model with mass degeneracies.

In this paper, we shall pursue several different goals:

1. Reliable MUED event generation. By now MUED is available in several parton level event generators. The original implementation [57] was done directly in CALCHEP [58] and COMPHEP [59], while the more modern implementations in MADGRAPH [60] and Whizard [61, 62] have interfaced to the FeynRules package [63]. Since parton level event generators exclusively define the final state at the parton level, they are ideally suited for simplified model studies, while complete models are traditionally more conveniently studied with general purpose event generators, which eventually have to be used as part of the simulation chain anyway. While MUED is available in Herwig ++ [64] and Sherpa [65], here we shall focus on the case of Pythia given its wide use in the experimental community. The original implementation [66-68] was done in the fortran version PyтHIA6 [69] which is now being phased out. One of our major goals here is to create a modern implementation of MUED in PYTHIA8 [70], as well as to validate and $\mathrm{fix}^{3}$ for backward compatibility the current PYTHIA6 version, which has a couple of issues. First, the matrix elements incorporated in PyтHIA were computed in the degenerate limit, ignoring the mass splittings among the KK partners at a given KK level. Here we will account for the correct mass splittings among the level $1 \mathrm{KK}$ partners. The corresponding analytical results are given in Appendix A, while in Section 3.1 we also quote the corresponding expressions for the degenerate mass limit, correcting some previous results in the literature. Second, we shall be careful to include all strong production subprocesses. Finally, we shall expand the nomenclature to allow separate treatment of KK partners with different gauge quantum numbers. The details of our Pyтнia6 (Pythia8) implementation can be found in Appendix B (Appendix C).

2. Estimate the systematic uncertainties in the UED mass spectrum calculation. Even though the formulas for the one-loop corrections to the KK mass spectrum have been known for a while [37], when implementing them in an event generator, one has to face several decisions which may affect the results. In the spirit of refs. [19, 20], in Section 2.2 we shall compare the MUED mass spectra resulting from the existing implementations in Pythia and CALCHEP and identify the origin of the observed differences.

\footnotetext{
${ }^{3}$ The history of the MUED implementation in PyTHIA6 is somewhat complicated. The first papers to calculate the matrix elements $[66,71]$ contained some typos, some of which were eventually fixed in an erratum several years later [72]. Unfortunately, by then the work on implementing UED in PYTHIA had already started [67] and those corrections did not propagate to the official version [68].
} 
3. Estimate the relative importance of level $2 K K$ resonances for the inclusive production of level $1 \mathrm{KK}$ particles. While our main focus will be on strong pair-production of level $1 \mathrm{KK}$ partners, in Section 3.3 we shall also evaluate the relative contributions from diagrams with level $2 \mathrm{KK}$ partners.

4. Apply Checkmate to the case of MUED. At the moment, there exist several packages which allow testing of BSM models against published LHC results, e.g. Checkmate [73], SModelS [74], Fastlim [75], Madanalysis5 [76], etc. However, most of the previous applications in the literature have been limited to some version of supersymmetry. Here we would like to showcase a different complete model, namely MUED, using the latest version of CHECKMATE [77].

5. Derive the current LHC bounds on minimal UED. Qualitatively, the collider signatures of MUED are similar to those of supersymmetry with conserved $R$-parity and a neutral LSP [78]. For example, for every simplified model event topology in SUSY, there is an analogous one in MUED. However, there are important quantitative differences as well. First, because of the relatively small mass splittings among the KK partners, the visible SM decay products tend to be rather soft, which would hurt the efficiency. This may be offset by the fact that the KK quarks are fermions, and thus have larger cross-sections compared to the spin 0 squarks in SUSY. Finally, the mass degeneracy of the KK partners implies that there are many relevant subprocesses, and therefore, a large number of final state signatures which one could potentially target. An important theoretical question, therefore, is what is the optimal search strategy for MUED in terms of the discovery signature. A tool like Checkmate is perfect to answer this question.

Previously there have been several related studies of MUED phenomenology at hadron colliders (see $[79,80]$ for reviews). In general, the main characteristic feature of the MUED collider signature depends on whether the lightest KK particle (LKP) is allowed to decay to SM particles through some sort of KK-parity violating interactions or not [81, 82]. Here we shall focus on the most challenging scenario in which the LKP is stable and neutral, as in supersymmetry, and consider pair-production of level $1 \mathrm{KK}$ partners ${ }^{4}$. Then the generic signal is missing transverse energy, accompanied by (typically soft) decay products from KK cascade decays. Given the softness of the signatures, the original papers focused at first on the clean leptonic channels, which represent golden channels for discovery [78, 90-94]. Subsequently, multilepton signatures with jets were also considered [95-97], and even hadronic multijet channels [98-100] were studied. In the extreme case of very small mass splittings, where none of the decay products can be reliably reconstructed, it has been suggested that one could usefully use a jet from initial state radiation [101] or analyze the pattern of reconstructed tracks [102]. In Section 4 we shall use the full suite of LHC analyses implemented in Checkmate to derive LHC limits on the MUED scale $R^{-1}$ from Run 1 data, which can

\footnotetext{
${ }^{4}$ Unlike the case of SUSY, in UED one could also look for the single production of level 2 KK resonances $[44,83-89]$, which is beyond the scope of this paper.
} 


\begin{tabular}{|c|c|c|c|}
\hline Level 1 particle & \multirow{2}{*}{ Notation } & \multicolumn{2}{|c|}{ Mass $(\mathrm{GeV})$} \\
\cline { 3 - 4 } & & CALCHEP & PYTHIA6 \\
\hline \hline KK gluon & $g_{1}^{\star}$ & 640.49 & 622.55 \\
\hline \hline KK doublet quark $(u$ - or $d$-type) & $q_{1}^{\bullet}$ & - & - \\
\hline KK doublet $u$ quark & $u_{1}^{\bullet}$ & 597.57 & 584.76 \\
\hline KK doublet $d$ quark & $d_{1}^{\bullet}$ & 597.57 & 584.76 \\
\hline \hline KK singlet quark $(u$ - or $d$-type) & $q_{1}^{\circ}$ & - & - \\
\hline KK singlet $u$ quark & $u_{1}^{\circ}$ & 586.24 & 574.23 \\
\hline KK singlet $d$ quark & $d_{1}^{\circ}$ & 584.42 & 572.53 \\
\hline \hline Generic KK quark & $q_{1}^{*}$ & - & - \\
\hline
\end{tabular}

Table 1. Notation for the colored level $1 \mathrm{KK}$ partners and their masses obtained with default settings from CALCHEP and Pythia for $R^{-1}=500 \mathrm{GeV}$ and $\Lambda R=20$.

be compared to published ATLAS results [103-106]. Such LHC results have interesting cosmological implications, since they test the idea that Kaluza-Klein dark matter is a viable component of the dark matter in the Universe [107]. Section 5 is reserved for our conclusions.

\section{MUED preliminaries}

\subsection{Notations and conventions}

To the extent that we are interested in LHC phenomenology, our focus here will be on the strongly interacting KK partners: the KK gluons, $g_{n}^{\star}$, and the KK quarks, $q_{n}^{\bullet}$ and $q_{n}^{\circ}$, where the index $n$ labels the KK level. We follow the notation of [71], where the KK excitation of an $S U(2)$-doublet fermion of the SM is denoted with a bullet (a filled circle), while the KK excitation of an $S U(2)$-singlet fermion of the SM is denoted with an open circle (see Table 1). If the $S U(2)$ quantum number of the KK quark could be either one, we shall use an asterisk as a placeholder.

While the SM quarks are chiral and receive their tree-level masses from the Higgs mechanism, the KK modes $q_{n}^{\bullet}$ and $q_{n}^{\circ}$ (with $n \geq 1$ ) have both a left-handed and a right-handed component, and obtain their masses predominantly from the compactification:

$$
m_{q_{n}^{*}}=\sqrt{\left(n R^{-1}\right)^{2}+m_{q_{0}}^{2}}, \quad \text { for } n \geq 1 .
$$

Since the KK modes at level 1 (i.e., with $n=1$ ) are the lightest, they are most easily produced at the LHC, and so will be the main focus of our discussion in the next Section. In MUED, KK parity is conserved, and level 1 gluons $g_{1}^{\star}$ and level 1 quarks $q_{1}^{*}$ are necessarily pair-produced. They will then cascade decay to the LKP, which in MUED is the level $1 \mathrm{KK}$ mode $B_{1}$ of the SM hypercharge gauge boson.

\subsection{Mass spectrum uncertainties}

Obtaining an accurate particle mass spectrum in a given new physics scenario is the first step in any robust phenomenological study. In MUED, all boundary terms are assumed to vanish 
at some matching scale $\Lambda>R^{-1}$, but get regenerated at lower scales $\mu$ from RGE running, which leads to a set of "boundary" mass corrections to the level 1 KK modes [37]

$$
\begin{aligned}
& \bar{\delta} m_{q_{n}^{\bullet}}=\frac{n}{R}\left(3 \frac{g_{3}^{2}}{16 \pi^{2}}+\frac{27}{16} \frac{g_{2}^{2}}{16 \pi^{2}}+\frac{1}{16} \frac{g^{\prime 2}}{16 \pi^{2}}\right) \ln \frac{\Lambda^{2}}{\mu^{2}}, \\
& \bar{\delta} m_{u_{n}^{\circ}}=\frac{n}{R}\left(3 \frac{g_{3}^{2}}{16 \pi^{2}}+\frac{g^{\prime 2}}{16 \pi^{2}}\right) \ln \frac{\Lambda^{2}}{\mu^{2}}, \\
& \bar{\delta} m_{d_{n}^{\circ}}=\frac{n}{R}\left(3 \frac{g_{3}^{2}}{16 \pi^{2}}+\frac{1}{4} \frac{g^{\prime 2}}{16 \pi^{2}}\right) \ln \frac{\Lambda^{2}}{\mu^{2}}, \\
& \bar{\delta} m_{g_{n}^{\star}}^{2}=\frac{n^{2}}{R^{2}} \frac{23}{2} \frac{g_{3}^{2}}{16 \pi^{2}} \ln \frac{\Lambda^{2}}{\mu^{2}} .
\end{aligned}
$$

In addition, the KK gluons also receive corrections from bulk loops [37]

$$
\delta m_{g_{n}^{\star}}^{2}=-\frac{3}{2} \frac{\zeta(3) g_{3}^{2}}{16 \pi^{4}} \frac{1}{R^{-2}},
$$

which are not logarithmically enhanced and are generally numerically quite small.

In the last two columns of Table 1 we show the mass spectrum for the level 1 colored KK partners which results from the application of the mass corrections (2.2-2.6) in the two packages CALCHEP and Pythia6, respectively. In both cases, we use default settings and input the same MUED parameters: $R^{-1}=500 \mathrm{GeV}$ and $\Lambda R=20$, yet the calculated masses are noticeably different. In particular, the mass spectrum obtained in PYTHIA6 appears to be slightly lighter. The origin of this difference can be tracked back to the way in which the two programs compute the values for the SM gauge couplings $\left(g_{3}, g_{2}, g^{\prime}\right)$ which enter eqs. (2.2-2.6) and the renormalization scale $\mu$ at which (2.2-2.6) are applied.

- In Pythia6, the MUED spectrum is computed in the PYUEDC subroutine. The running strong coupling $g_{3}(Q)$ is evaluated at the scale $Q=R^{-1}$ through the Pythia function PYALPS, using the default $\Lambda_{Q C D}(=250 \mathrm{MeV})$ and a lowest order (LO) running. However, the electroweak couplings are not evolved, and PYUEDC uses a fixed input $\alpha_{e m}=1 / 137$ through the default setting of the parameter PARU(101). The values of $g_{2}$ and $g^{\prime}$ which are needed in (2.2-2.4) are then derived from the fixed inputs for $\alpha_{e m}$ and the Weinberg angle, $\theta_{W}$.

- In contrast, the MUED formulation for CALCHEP runs either all three couplings (with the parameter $R G$ set to 1 ) or none (with $R G=0$, which is the default setting). For the case of running couplings, the scale $\mu$ at which the couplings are evaluated is set by the parameter scaleN: with $\mathrm{scaleN}=1$, it is set at $\mu=R^{-1}$, while scaleN $=2$ (the default) implies $\mu=2 R^{-1}$. When $\mathrm{RG}=0$, the three gauge couplings $\alpha_{i}$ are fixed at their values at $\mu=M_{Z}$ through the input variables c1MZ, c2MZ and c3MZ in CalcHEP [57].

It is clear that by default, not all three gauge couplings are consistently run to the same renormalization scale in the two programs. In principle, the differences are higher order, and are expected to be of the same size as the neglected next-to-leading order corrections in (2.2-2.6). As a sanity check, we have confirmed that when the gauge couplings are treated the same way, i.e. 
- we let all three gauge couplings run at the same time,

- we use the same starting values at $M_{Z}$,

- the running is done at leading order,

- the couplings are evolved up to the same renormalization scale $\mu=R^{-1}$,

the two programs give identical results for the masses of the level $1 \mathrm{KK}$ particles. This exercise required appropriate tweaking of the code and/or some of the program parameters. Note that the choice of renormalization scale $\mu$ used for the computation of the KK mass spectrum may in principle be different from the choice of $\mu$ used for the gauge couplings appearing in the scattering (decay) vertices. Those couplings need to be evaluated at a scale compatible with the set-up for the hard-scattering, particularly since the parton distribution in use provides the value of $\alpha_{s}$ compatible with its parametrization.

In summary, the differences seen in Table 1 between the default mass spectrum calculations in PYTHIA and CALCHEP are due to the effect from higher order terms, and are thus indicative of the theoretical uncertainty of these calculations. In what follows, we shall sometimes need to compare cross-section results from PYTHIA and CALCHEP for the same KK spectrum. To do so, for concreteness we shall first obtain the default PYTHIA mass spectrum and then feed those masses to CALCHEP, rather than the other way around - this is easier operationally, since in CALCHEP the UED masses can be easily set externally, without hacking the internal code.

\section{Strong production in MUED}

In this section we discuss strong production in pure QCD of level $1 \mathrm{KK}$ partners and validate its implementation in CALCHEP and PythiA. At parton level, these processes were first computed in [71] in the degenerate limit

$$
m_{g_{1}^{\star}}=m_{q_{1}^{*}} \equiv m_{K},
$$

where one neglects the mass differences among the different level $1 \mathrm{KK}$ quarks and/or the KK gluon. The calculation was repeated in Ref. [66], whose analytical results were then used for the MUED implementation in Pyтнia6 [67, 68]. Since the mass splittings among the level $1 \mathrm{KK}$ partners arise from renormalization effects, (3.1) is not a bad approximation, but we would like to avoid it nevertheless. Thus we have revisited the calculation of the parton-level pair production cross-sections for various pairs of colored level $1 \mathrm{KK}$ partners in the general case of arbitrary mass splittings. The corresponding results are listed in Appendix A. The formulas are rather long and not very instructive, but we shall make use of them later on when we create our own MUED implementation in PyTHIA6, as explained in Appendix B. Here (in Section 3.1) we quote the simplified version of our results from Appendix A in the degenerate limit (3.1), which can be compared with the previous literature. This will also allow us to test the current version of Pythia6 in Section 3.2. Finally, in Section 3.3 we close this section with a comment on the relative importance of diagrams mediated by level 2 KK particles. 


\subsection{Analytical results in the limit of degenerate level $1 \mathrm{KK}$ partners}

In this subsection we list the simplified version of our results from Appendix A in the degenerate limit (3.1). The formulas are written in terms of the Mandelstam variables $s, t$ and $u$. In the degenerate case (3.1), the two final state particles have the same mass $m_{K}$, and the Mandelstam variables obey the identity

$$
s+t+u=2 m_{K}^{2} .
$$

This is why it is convenient to redefine

$$
\begin{aligned}
t^{\prime} & \equiv t-m_{K}^{2}, \\
u^{\prime} & \equiv u-m_{K}^{2},
\end{aligned}
$$

after which eq. (3.2) simplifies to

$$
s+t^{\prime}+u^{\prime}=0
$$

The spin-averaged squared matrix elements for the different $2 \rightarrow 2$ strong production processes are given by

$$
\begin{aligned}
& \bar{\sum}|\mathcal{M}|_{g g \rightarrow g_{1}^{\star} g_{1}^{\star}}^{2}=\frac{9 \alpha_{s}^{2}}{16\left(s t^{\prime} u^{\prime}\right)^{2}}\left(s^{2}+t^{\prime 2}+u^{\prime 2}\right) \\
& \quad \times\left[6 m_{K}^{4} s^{2}-6 m_{K}^{2} s t^{\prime} u^{\prime}+3 t^{\prime 2} u^{\prime 2}+2 s^{2}\left(t^{\prime 2}+u^{\prime 2}\right)\right] \\
& \bar{\sum}|\mathcal{M}|_{q g \rightarrow q_{1}^{*} g_{1}^{\star}}^{2}=\frac{-\alpha_{s}^{2}}{72 s t^{\prime} u^{\prime 2}}\left(2 s^{2}+2 t^{\prime 2}+u^{\prime 2}\right)\left(9 s^{2}+9 t^{\prime 2}-u^{\prime 2}\right) \\
& \bar{\sum}|\mathcal{M}|_{q q \rightarrow q_{1}^{*} q_{1}^{*}}^{2}=\frac{\alpha_{s}^{2}}{54 t^{\prime 2} u^{\prime 2}}\left[t^{4}+u^{\prime 4}-s^{4}+6 s^{2}\left(t^{\prime 2}+u^{\prime 2}\right)+m_{K}^{2}\left(6 t^{\prime 3}+6 u^{\prime 3}-s t^{\prime} u^{\prime}\right)\right] \\
& \bar{\sum}|\mathcal{M}|_{q q^{\prime} \rightarrow q_{1}^{*} q_{1}^{\prime *}}^{2}=\frac{\alpha_{s}^{2}}{18 t^{\prime 2}}\left(-4 m_{K}^{2} s+4 s^{2}+t^{\prime 2}\right) \\
& \bar{\sum}|\mathcal{M}|_{g g \rightarrow q_{1}^{*} \bar{q}_{1}^{*}}^{2}=\frac{-\alpha_{s}^{2}}{24 s^{2} t^{\prime 2} u^{\prime 2}}\left(4 s^{2}-9 t^{\prime} u^{\prime}\right)\left[4 m_{K}^{4} s^{2}-4 m_{K}^{2} s t^{\prime} u^{\prime}-u^{\prime} t^{\prime}\left(t^{\prime 2}+u^{\prime 2}\right)\right] \\
& \bar{\sum}|\mathcal{M}|_{q \bar{q} \rightarrow q_{1}^{*} \bar{q}_{1}^{*}}^{2}=\frac{\alpha_{s}^{2}}{54 s^{2} t^{\prime 2}}\left[12 m_{K}^{2} s\left(s^{2}-s t^{\prime}+4 t^{\prime 2}\right)\right. \\
& \left.\quad+12 s^{4}+16 s^{3} t^{\prime}+23 s^{2} t^{\prime 2}+36 s t^{\prime 3}+48 t^{\prime 4}\right] \\
& \bar{\sum}|\mathcal{M}|_{q \bar{q}^{\prime} \rightarrow q_{1}^{*} \bar{q}_{1}^{\prime \circ}}^{2}=\frac{\alpha_{s}^{2}}{18 t^{\prime 2}}\left(-4 m_{K}^{2} s+4 s^{2}+t^{\prime 2}\right) \\
& \bar{\sum}|\mathcal{M}|_{q \bar{q}^{\prime} \rightarrow q_{1}^{*} \bar{q}_{1}^{\prime *}}^{2}=\frac{\alpha_{s}^{2}}{18 t^{\prime 2}}\left(4 m_{K}^{2} s+4 s^{2}+8 s t^{\prime}+5 t^{\prime 2}\right) \\
& \bar{\sum}|\mathcal{M}|_{q q \rightarrow q_{1}^{*} q_{1}^{\circ}}^{2}=\frac{\alpha_{s}^{2}}{9 t^{\prime 2} u^{\prime 2}}\left[2 m_{K}^{2} s\left(t^{\prime 2}+u^{\prime 2}\right)+2 s^{4}-8 s^{2} t^{\prime} u^{\prime}+5 t^{\prime 2} u^{\prime 2}\right]
\end{aligned}
$$




$$
\begin{aligned}
& \bar{\sum}|\mathcal{M}|_{q q^{\prime} \rightarrow q_{1}^{*} q_{1}^{\prime \circ}}^{2}=\frac{\alpha_{s}^{2}}{18 t^{\prime 2}}\left(4 m_{K}^{2} s+4 s^{2}+8 s t^{\prime}+5 t^{\prime 2}\right), \\
& \bar{\sum}|\mathcal{M}|_{q \bar{q} \rightarrow q_{1}^{\prime *} \bar{q}_{1}^{\prime *}}^{2}=\frac{4 \alpha_{s}^{2}}{9 s^{2}}\left(2 m_{K}^{2} s+t^{\prime 2}+u^{\prime 2}\right), \\
& \bar{\sum}|\mathcal{M}|_{q \bar{q} \rightarrow g_{1}^{\star} g_{1}^{\star}}^{2}=\frac{-\alpha_{s}^{2}}{27 s^{2} t^{\prime 2} u^{\prime 2}}\left(4 s^{2}-9 t^{\prime} u^{\prime}\right)\left[\left(5 s^{2}-12 t^{\prime} u^{\prime}\right)\left(m_{K}^{2} s-t^{\prime} u^{\prime}\right)-4 m_{K}^{2} s^{3}\right] .
\end{aligned}
$$

Several sanity checks of these formulas are possible. Each expression has a prefactor whose denominator indicates the type of relevant diagrams. For example, one can verify that processes $(3.6),(3.7),(3.10)$ and (3.17) are mediated by $s^{-}, t$ - and $u$-channel diagrams; processes (3.8) and (3.14) have $t$ - and $u$-channel contributions; process (3.11) has $s$ - and $t$ channel diagrams; processes (3.9), (3.12), (3.13) and (3.15) are mediated by a single $t$-channel diagram, while (3.16) is a pure $s$-channel process. Second, processes with identical initial or final state particles should be invariant under $u \leftrightarrow t$. It is easy to see that the respective processes (3.6), (3.8), (3.10), (3.14) and (3.17) indeed obey this condition.

\subsection{Pythia6 validation}

The parton-level results (3.6-3.16) from the previous subsection can be used to test the Pythia6 implementation ${ }^{5}$ of MUED $[67,68]$ which was done in the same degenerate limit (3.1). To be more precise, in Pythia6 the approximation (3.1) is applied only when computing the event weight in the PYXUED subroutine, which introduces a common mass variable xmnkk for all level $1 \mathrm{KK}$ particles (the same as the common mass $m_{K}$ used in eqs. (3.6-3.17) above). On the other hand, the treatment of the phase-space weights in the Pythia subroutine PYSIGH correctly accounts for the actual masses of the individual KK excitations as calculated in the PYUEDC subroutine. Thus, there appears to be an inconsistency in the way these two basic parts of the Pyтнia6 code operate, and our goal in this subsection will be to quantify the effect and ultimately rectify this inconsistency. Along the way, we shall also validate the programmed matrix elements in PYтніA6 and perform the necessary corrections.

In Table 2 we compare $7 \mathrm{TeV}$ LHC cross-section results from the default MUED implementations in CALCHEP [57] (fourth column) and in Pythia6 [67, 68] (fifth column) on a process by process basis. In each case, we use the same KK mass spectrum as calculated in Pythia6 for $R^{-1}=500 \mathrm{GeV}, \Lambda R=20$. The first two columns identify the parton level subprocess, which in PYTHIA6 is labelled by the ISUB variable. The second column of Table 2 lists the generic subprocess. For ease of comparison, we do not sum over quark flavors, but choose a specific flavor selection as indicated in the table. In general, the full matrix element for each subprocess (see Appendix A) carries dependence on the masses of one or more KK particles, as indicated in the third column of Table 2. In both Pythia6 and CalcHEP we use CTEQ5L parton distribution functions and use the value of $\alpha_{s}$ returned by setting the renormalization scale $Q^{2}=\hat{s}$, which is the default in CALCHEP.

\footnotetext{
${ }^{5}$ The process of eq. (3.17), $q \bar{q} \rightarrow g_{1}^{\star} g_{1}^{\star}$, was not included in PyTHiA6 since at the LHC it was expected to have a relatively small cross-section in comparison to $g g \rightarrow g_{1}^{\star} g_{1}^{\star}$ of eq. (3.6).
} 


\begin{tabular}{|c|c|c|c|c|c|c|c|}
\hline \multirow{2}{*}{\multicolumn{2}{|c|}{$\begin{array}{c}\text { Parton-level } \\
\text { subprocess }\end{array}$}} & \multirow{3}{*}{$\begin{array}{c}\text { Mass } \\
\text { parameters }\end{array}$} & \multirow{3}{*}{$\begin{array}{c}\text { CALCHEP } \\
\sigma(\mathrm{fb})\end{array}$} & \multicolumn{4}{|c|}{ PYTHIA6 } \\
\hline & & & & \multirow{2}{*}{$\begin{array}{c}\text { default } \\
\sigma(\mathrm{fb})\end{array}$} & \multirow{2}{*}{$\begin{array}{c}\text { diff } \\
\%\end{array}$} & \multirow{2}{*}{$\begin{array}{c}\text { modified } \\
\sigma(\mathrm{fb})\end{array}$} & \multirow{2}{*}{$\begin{array}{c}\text { diff } \\
\%\end{array}$} \\
\hline ISUB & name & & & & & & \\
\hline 311 & $g g \rightarrow g_{1}^{\star} g_{1}^{\star}$ & $m_{g_{1}^{\star}}$ & 783.38 & 810.34 & 3.44 & 787.7 & 0.55 \\
\hline 312 & $\begin{aligned}(q g & \left.\rightarrow q_{1}^{*} g_{1}^{\star}\right) \\
u g & \rightarrow u_{1}^{\bullet} g_{1}^{\star}\end{aligned}$ & $m_{q_{1}^{*}}, m_{g_{1}^{\star}}$ & 1040.3 & 1893.36 & 82.0 & 1057 & 1.59 \\
\hline $\begin{array}{l}313 a \\
313 b\end{array}$ & $\begin{aligned}(q q & \left.\rightarrow q_{1}^{*} q_{1}^{*}\right) \\
u u & \rightarrow u_{1}^{\bullet} u_{1}^{\bullet} \\
\left(q q^{\prime}\right. & \left.\rightarrow q_{1}^{*} q_{1}^{\prime *}\right) \\
u d & \rightarrow u_{1}^{\bullet} d_{1}^{\bullet}\end{aligned}$ & $\begin{array}{c}m_{q_{1}^{*}}, m_{g_{1}^{\star}} \\
m_{q_{1}^{*}}, m_{q_{1}^{\prime *}}, m_{g_{1}^{\star}}\end{array}$ & $\begin{array}{l}549.6 \\
288.8\end{array}$ & $\begin{array}{r}1258.23 \\
551.70\end{array}$ & $\begin{array}{l}128.94 \\
91.03\end{array}$ & $\begin{array}{l}752.3 \\
295.2\end{array}$ & $\begin{array}{l}31.14 \\
2.19\end{array}$ \\
\hline 314 & $\begin{aligned}(g g & \left.\rightarrow q_{1}^{*} \bar{q}_{1}^{*}\right) \\
g g & \rightarrow u_{1}^{\bullet} \bar{u}_{1}^{\bullet}\end{aligned}$ & $m_{q_{1}^{*}}$ & 14.78 & 6.7 & 54.67 & 13.88 & 6.28 \\
\hline 315 & $\begin{array}{c}\left(q \bar{q} \rightarrow q_{1}^{*} \bar{q}_{1}^{*}\right) \\
u \bar{u} \rightarrow u_{1}^{\bullet} \bar{u}_{1}^{*}\end{array}$ & $m_{q_{1}^{*}}, m_{g_{1}^{\star}}$ & 47.81 & 82.14 & 71.80 & 58.5 & 20.11 \\
\hline 316 & $\begin{aligned}\left(q \bar{q}^{\prime}\right. & \left.\rightarrow q_{1}^{\bullet} \bar{q}_{1}^{\prime \circ}\right) \\
u \bar{d} & \rightarrow u_{1}^{\bullet} \bar{d}_{1}^{\circ}\end{aligned}$ & $m_{q_{1}^{\bullet}}, m_{q_{1}^{\prime \circ}}, m_{g_{1}^{\star}}$ & 52.03 & 85.13 & 63.62 & 53.25 & 2.31 \\
\hline 317 & $\begin{array}{c}\left(q \bar{q}^{\prime} \rightarrow q_{1}^{*} \bar{q}_{1}^{\prime *}\right) \\
u \bar{d} \rightarrow u_{1}^{\bullet} \bar{d}_{1}^{\bullet}\end{array}$ & $m_{q_{1}^{*}}, m_{q_{1}^{\prime *}}, m_{g_{1}^{\star}}$ & 34.92 & 57.97 & 66.01 & 36.37 & 4.07 \\
\hline $\begin{array}{l}318 \mathrm{a} \\
318 \mathrm{~b}\end{array}$ & $\begin{aligned}(q q & \left.\rightarrow q_{1}^{\bullet} q_{1}^{\circ}\right) \\
u u & \rightarrow u_{1}^{\bullet} u_{1}^{\circ} \\
\left(q q^{\prime}\right. & \left.\rightarrow q_{1}^{\bullet} q_{1}^{\prime \circ}\right) \\
u d & \rightarrow u_{1}^{\bullet} d_{1}^{\circ}\end{aligned}$ & $\begin{array}{l}m_{q_{1}^{\bullet}}, m_{q_{1}^{\circ}}, m_{g_{1}^{\star}} \\
m_{q_{1}^{\bullet}}, m_{q_{1}^{\prime \circ}}, m_{g_{1}^{\star}}\end{array}$ & 1027.8 & $\begin{array}{r}1474.94 \\
353.12\end{array}$ & $\begin{array}{l}43.50 \\
66.88\end{array}$ & 218.5 & $\begin{array}{l}1.73 \\
3.2\end{array}$ \\
\hline 319 & $\begin{array}{c}\left(q \bar{q} \rightarrow q_{1}^{\prime *} \bar{q}_{1}^{\prime *}\right) \\
u \bar{u} \rightarrow d_{1}^{\bullet} \bar{d}_{1}^{\bar{*}}\end{array}$ & $m_{q_{1}^{\prime *}}$ & 13.87 & 12.52 & 9.73 & 13.85 & 0.14 \\
\hline
\end{tabular}

Table 2. Comparison of the MUED cross sections for different subprocesses at the $7 \mathrm{TeV} \mathrm{LHC}$, obtained with two different MUED implementations: CALCHEP [57] and Pythia6 [67, 68]. In both programs, we choose the CTEQ5L parton distribution functions with $Q^{2}=\hat{s}$ and use the default mass spectrum as calculated in PYтнIA with $R^{-1}=500 \mathrm{GeV}, \Lambda R=20$. The first two columns identify the parton level subprocess, while the third column lists the mass parameters which enter the corresponding full matrix element (see Appendix A). The fourth (fifth) column gives the default result from CALCHEP (Pyтhia6), and the sixth column gives the percent difference between them. The last two columns give the corresponding results from a similar comparison with a modified version of PYTHIA6 with a suitable choice of the parameter xmnkk as explained in the text.

The sixth column of Table 2 displays the percent difference of the results. We notice that the default answers from CALCHEP and PYTHIA6 can be quite different, which prompts an investigation into the potential origin of this mismatch. Since we have matched the mass spectra by hand, we have already ruled out the mass uncertainties discussed previously in Section 2.2.

The main culprit for the discrepancy is the use of the degenerate mass approximation (3.1). By design, the MUED matrix elements in PYTHIA were programmed in the degenerate mass limit (3.1), which was adopted in Refs. [66, 71]. In contrast, the matrix elements in CALCHEP are computed with the correct mass spectrum, including the proper 1-loop mass 
splittings. We also note that the common KK mass xmnkk in PYтнIA by default is set equal to the mass of the lightest KK particle, the level $1 \mathrm{KK}$ mode of the hypercharge gauge boson, $B_{1}$. This value is then used to compute the cross-sections for all subprocesses, including strong production. In reality, the scale for the strong production cross-sections is set by the masses of the colored level $1 \mathrm{KK}$ partners which are heavier than $B_{1}$ by $10-20 \%$ [37]. This would lead to a systematic overestimation of the strong cross-sections in PүтнIA, which is indeed what we observe ${ }^{6}$ in Table 2.

Having identified the problem, the question now is whether one can still recover the correct answer in PYTHIA without reprogramming the code with the full matrix elements from Appendix A, and instead simply making a judicious choice of the value of the common mass xmnkk. This seems to be plausible in the case of subprocesses where the full matrix element depends on a single mass parameter, namely the mass of the (identical) final state particles. Table 2 contains three such processes, ISUB $=\{311,314,319\}$.

Let us illustrate the procedure with the example of KK gluon pair production where the matrix element depends on a single parameter, $m_{g_{1}^{\star}}$. If we substitute $m_{K}=m_{g_{1}^{\star}}$ in the degenerate case formula (3.6), we obtain

$$
\begin{aligned}
\bar{\sum}|\mathcal{M}|_{g g \rightarrow g^{\star} g^{\star}}^{2}=\frac{9 \alpha_{s}^{2}}{16\left(s t^{\prime} u^{\prime}\right)^{2}}\left(s^{2}+t^{\prime 2}+u^{\prime 2}\right) \\
\quad \times\left[6 m_{g_{1}^{\star}}^{4} s^{2}-6 m_{g_{1}^{\star}}^{2} s t^{\prime} u^{\prime}+3 t^{\prime 2} u^{\prime 2}+2 s^{2}\left(t^{\prime 2}+u^{\prime 2}\right)\right],
\end{aligned}
$$

where now $t^{\prime}=t-m_{g_{1}^{\star}}^{2}$ and $u^{\prime}=u-m_{g_{1}^{\star}}^{2}$. The result is identical to eq. (A.1), which holds in the non-degenerate limit. This demonstrates that for single-parameter matrix elements, we should be able to recover the correct answer with the proper choice of the parameter xmnkk in PyтнiA. The last two columns in Table 2 show that this trick generally works: after modifying the default choice for xmnkk, we get excellent agreement for two out of the three processes, namely ISUB $=311$ and ISUB $=319$. On the other hand, the discrepancy for ISUB $=314$ is still sizable, on the order of $6 \%$, and deserves further scrutiny. The subprocess is $g g \rightarrow q_{1}^{*} \bar{q}_{1}^{*}$, thus we need to replace $m_{K} \rightarrow m_{q_{1}^{*}}$ everywhere in (3.10), obtaining

$$
\bar{\sum}|\mathcal{M}|_{g g \rightarrow q_{1}^{*} \bar{q}_{1}^{*}}^{2}=\frac{-\alpha_{s}^{2}}{24 s^{2} t^{\prime 2} u^{\prime 2}}\left(4 s^{2}-9 t^{\prime} u^{\prime}\right)\left[4 m_{q_{1}^{*}}^{4} s^{2}-4 m_{q_{1}^{*}}^{2} s t^{\prime} u^{\prime}-u^{\prime} t^{\prime}\left(t^{\prime 2}+u^{\prime 2}\right)\right],
$$

which is the same answer as (A.5), and the simple fix above should have been sufficient. Due to the identical final state particles, the expression (A.5) is symmetric with respect to $u \leftrightarrow t$, but as it turns out, the PYThIA6 code is not. This indicates a problem, which was apparently inherited from the expressions in Ref. [66].

For processes whose matrix elements depend on 2 or 3 mass parameters (see the third column in Table 2), a valid procedure for lifting the degenerate limit expressions (3.6-3.17) to their non-degenerate counterparts from Appendix A is not available. Short of implementing

\footnotetext{
${ }^{6}$ There are two exceptions to this trend: the process $q \bar{q} \rightarrow q_{1}^{\prime *} \bar{q}_{1}^{\prime *}$ with ISUB $=319$, whose cross-section scales as $1 / s$ instead of $1 / m_{K}^{2}$, see (3.16), and the process $g g \rightarrow q_{1}^{*} \bar{q}_{1}^{*}$ with ISUB $=314$, for which the PYTHIA6 code contains a typo, as we will discuss below.
} 


\begin{tabular}{|c|c|c|c|c|}
\hline \multirow[b]{2}{*}{ ISUB } & \multirow[b]{2}{*}{ Process } & РҮтніА6 & PYTHIA8 & \multirow{2}{*}{$\frac{\text { CALCHEP }}{\text { matched spectrum }}$} \\
\hline & & \multicolumn{2}{|c|}{$R^{-1}=1 \mathrm{TeV}$} & \\
\hline 311 & $g g \rightarrow g_{1}^{\star} g_{1}^{\star}$ & 161.69 & 161.70 & 161.96 \\
\hline 312 & $q g \rightarrow q_{1}^{\bullet} g_{1}^{\star}+c . c$. & 644.59 & 644.90 & 645.79 \\
\hline 313 & $q g \rightarrow q_{1}^{\circ} g_{1}^{\star}+c . c$. & 675.13 & 675.9 & 676.89 \\
\hline 314 & $q^{i} q^{j} \rightarrow q_{1}^{\bullet i} q_{1}^{\bullet j}+$ c.c. & 285.90 & 285.30 & 285.84 \\
\hline 315 & $q^{i} q^{j} \rightarrow q_{1}^{\circ i} q_{1}^{\circ j}+$ c.c. & 303.28 & 303.80 & 303.71 \\
\hline 316 & $g g \rightarrow q_{1}^{\bullet} \bar{q}_{1}^{\bullet}$ & 14.69 & 14.75 & 14.71 \\
\hline 317 & $g g \rightarrow q_{1}^{\circ} \bar{q}_{1}^{\circ}$ & 17.34 & 17.37 & 17.36 \\
\hline 318 & $q^{i} \bar{q}^{j} \rightarrow q_{1}^{\bullet i} \bar{q}_{1}^{\bullet j}$ & 68.93 & 68.82 & 68.93 \\
\hline 319 & $q^{i} \bar{q}^{j} \rightarrow q_{1}^{\circ i} \bar{q}_{1}^{\circ j}$ & 75.01 & 75.23 & 75.05 \\
\hline 320 & $q^{i} \bar{q}^{j} \rightarrow q_{1}^{\bullet i} \bar{q}_{1}^{\circ j}$ & 163.98 & 164.5 & 164.41 \\
\hline 321 & $q^{i} q^{j} \rightarrow q_{1}^{\bullet i} q_{1}^{\circ j}+$ c.c. & 473.71 & 474.3 & 474.43 \\
\hline 322 & $q \bar{q} \rightarrow q_{1}^{\bullet \prime} \bar{q}_{1}^{\bullet \prime}$ & 35.24 & 35.29 & 35.32 \\
\hline 323 & $q \bar{q} \rightarrow q_{1}^{\circ \prime} \bar{q}_{1}^{\circ \prime}$ & 39.91 & 40.20 & 40.20 \\
\hline 324 & $q \bar{q} \rightarrow g_{1} g_{1}$ & 20.28 & 20.40 & 20.39 \\
\hline
\end{tabular}

Table 3. Comparison of the strong production cross-sections (in $\mathrm{fb}$ ) for different subprocesses in MUED at the $14 \mathrm{TeV}$ LHC. Results are shown for our MUED implementations in PYTHIA6 and Pythia8, as well as for CALCHEP with an identical KK mass spectrum. In all cases, we choose the CTEQ5L parton distribution functions from the LHAPDF v5.9.1 library [108], with $Q^{2}=\hat{s}$, and use the default mass spectrum as calculated in Pythia6 with $R^{-1}=1 \mathrm{TeV}$ and $\Lambda R=20$.

the full expressions from Appendix A, the best one could do is the following: in the denominators of the prefactors, replace $m_{K}$ with the mass of the corresponding KK particle in the $t$-channel and $u$-channel propagator, while in the remaining expressions use the average mass of the final state particles in place of $m_{K}$. Having made these corrections in Pythin6, we obtained the cross-sections listed in the second-to-last column of Table 2. Despite the ad hoc procedure used, the results are pretty close to the full answer from CALCHEP. There are two notable exceptions, the processes $313 \mathrm{a}$ and 315 . We have checked that again the discrepancies are due to typos inherited from Ref. [66] and left uncorrected.

Having identified the problems with the current implementation of MUED in PyThIA6, we modified the Pythia6 fortran code as explained in Appendix B. In addition to fixing the typos, we generalized the treatment of the KK quarks, allowing for the KK doublets $q_{1}^{\bullet}$ and singlets $q_{1}^{\circ}$ to be handled independently. Since the currently supported PYTHIA distribution is the $\mathrm{C}++$ Pythia8, we also provide an implementation of MUED in Pythia 8 which is described in Appendix C. Table 3 provides a numerical cross-check that the results obtained with CalcHEP [57] and with our two new versions of PYTHIA6 and PYTHIA8 are in agreement. Notice that since we now differentiate between KK doublets $q_{1}^{\bullet}$ and KK singlets $q_{1}^{\circ}$, the number of subprocesses in Table 3 is larger than what we had previously in Table 2. For example, the generic $q_{1}^{*} g_{1}^{\star}$ associated production ISUB $=312$ from Table 2 is now divided into the production of KK doublet quarks $q_{1}^{\bullet} g_{1}^{\star}\left(\right.$ ISUB=312) and KK singlet quarks $q_{1}^{\circ} g_{1}^{\star}($ ISUB=313) in Table 3. 

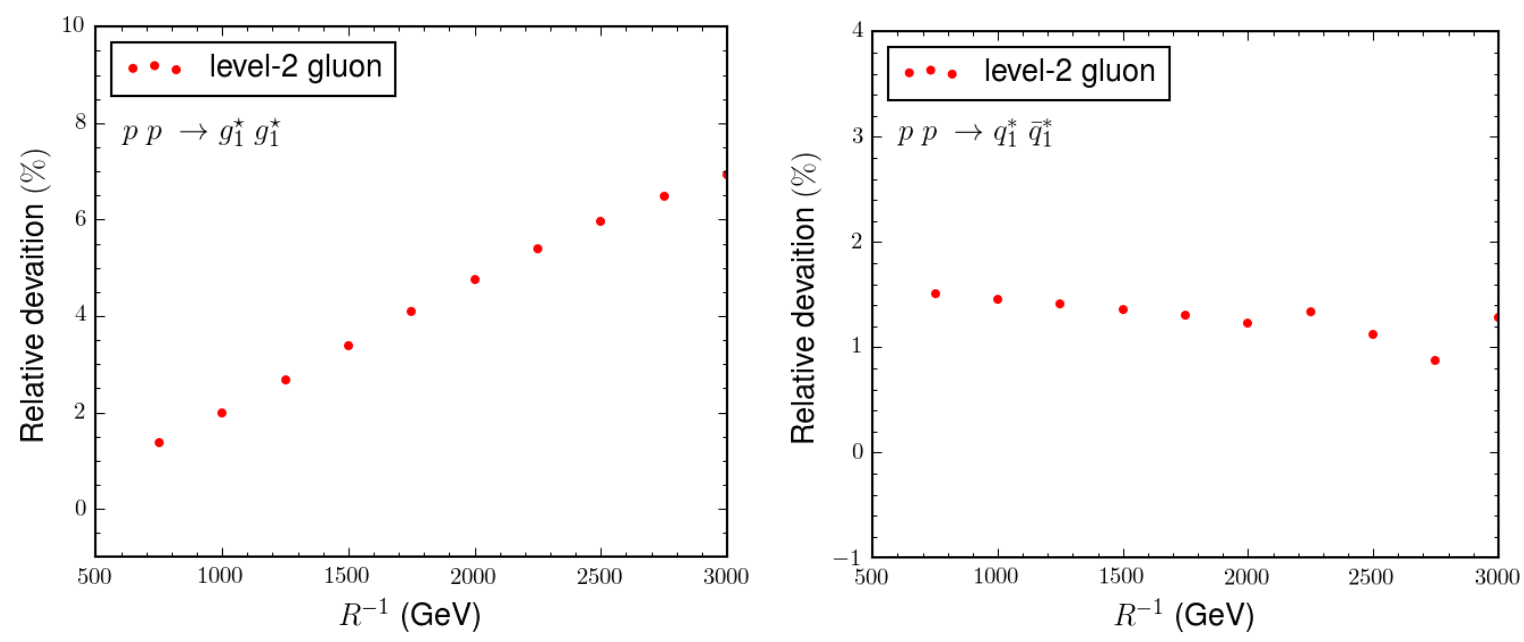

Figure 1. Relative deviation (in percent) of the cross-section for KK gluon pair-production (left) and KK quark pair-production, summed over all flavors, (right) after including $s$-channel propagators with virtual level 2 gluons. Results are plotted as a function of $R^{-1}$, for $\Lambda R=20, E_{c m}=14 \mathrm{TeV}$, and choosing the CTEQ5L PDF set with $Q^{2}=\hat{s}$.

\subsection{The relative importance of virtual level 2 KK particles}

The results (3.6-3.17) were derived ignoring diagrams containing $s$-channel propagators with level $2 \mathrm{KK}$ particles, since those always involve KK-parity conserving, but KK-number violating couplings between two SM particles and a level $2 \mathrm{KK}$ particle. Such couplings are suppressed, since they are generated at one loop, and thus one might expect the corresponding contributions to be relatively small.

Fig. 1 illustrates the relative importance of diagrams with virtual level $2 \mathrm{KK}$ gluons, for the case of KK gluon pair-production (left panel) and KK quark pair-production, summed over all flavors, (right panel). The results, obtained with CALCHEP, are plotted as a function of $R^{-1}$, for $\Lambda R=20$, center-of-mass energy $E_{c m}=14 \mathrm{TeV}$, and choosing the CTEQ5L PDF set. We see that the effect is at the order of a few percent, in accordance with expectations.

\section{Constraints on MUED}

\subsection{Constraints from cosmology}

Due to the conservation of KK parity, the LKP is stable and could be a dark matter candidate, as long as it is not charged or colored. It would then inherit all the attractive features of a generic WIMP, and can be probed both at colliders and in dark matter experiments [109]. MUED is a very restricted model, with only two parameters: $R^{-1}$ and $\Lambda$. The relic density of the LKP depends mostly on the mass scale of the LKP, $R^{-1}$, and less on $\Lambda$, which enters only logarithmically. Therefore, the requirement for the correct dark matter relic abundance singles out a preferred range for $R^{-1}$, setting a well-motivated target for the experimental searches. The close mass degeneracy of the level $1 \mathrm{KK}$ partners, however, 


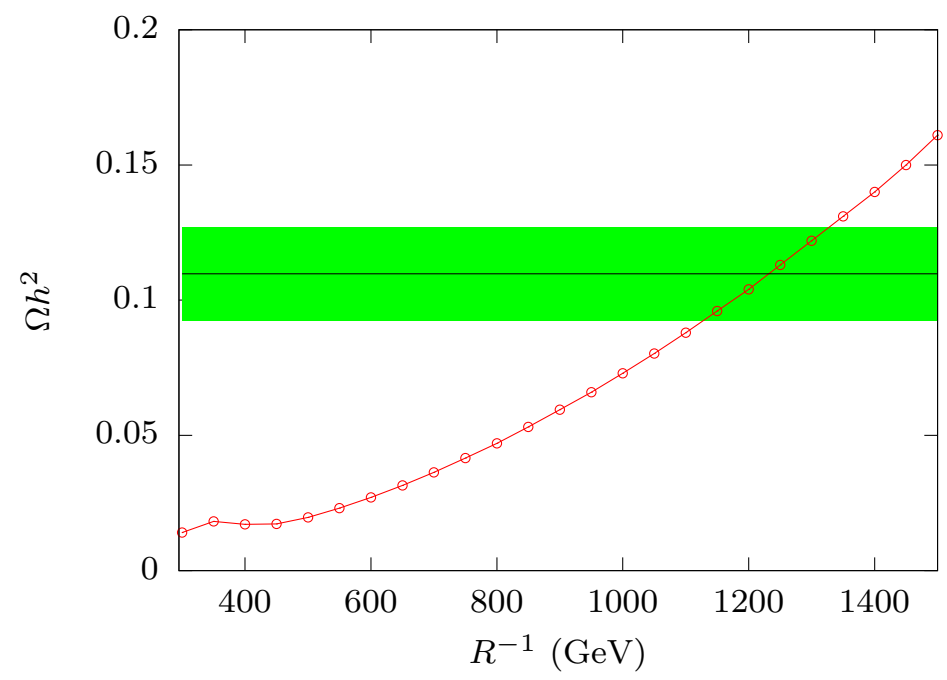

Figure 2. The relic density in MUED as a function of $R^{-1}$, for $\Lambda R=10$, as calculated with MICROMEGAs [116], using the the MUED model files from CALCHEP [57]. The horizontal green band indicates the $3 \sigma$ experimentally preferred range [117].

complicates the thermal freeze-out calculation, since one has to account for coannihilations. The very first calculation of the MUED relic density [110] considered coannihilations with the level 1 KK-leptons, which are closest in mass to the LKP. Subsequently, the full set of coannihilation processes were also included [111, 112], and the preferred mass range for the LKP (and therefore, for $R^{-1}$ ) was found to be on the order of $500-600 \mathrm{GeV}$. However, these calculations did not include contributions from diagrams with virtual level-2 KK particles, see Section 3.3. Although the direct couplings of two SM particles to a level-2 KK partner are loop suppressed, there is an $s$-channel resonant enhancement, and the limit on $R^{-1}$ is raised to over $1 \mathrm{TeV}[113,114]$. The latest state of the art calculation of the relic density in UED was done in Ref. [115], considering the KK photon $\gamma_{1}$ as LKP (and not the hypercharge gauge boson $B_{1}$ as in MUED). The preferred range was found to be in the neighborhood of $R^{-1} \sim 1.3-1.5 \mathrm{TeV}$, depending on the exact value of $\Lambda R$ and accounting for the astrophysics uncertainties.

The cosmological constraint on MUED is illustrated in Fig. 2, where we plot the $B_{1}$ relic density as a function of $R^{-1}$, for $\Lambda R=10$. We have used MICROMEGAs [116] with the MUED model files from the CALCHEP implementation [57]. We see from Fig. 2 that in MUED, the correct amount of dark matter is obtained for $R^{-1} \sim 1250 \mathrm{GeV}$. One might expect the true limit to be slightly higher, since the plot does not include contributions from level 2 Higgs $s$-channel resonances, which are not present in the model files from Ref. [57]. The bound on $R^{-1}$ can slightly be raised further, if we allow for higher values for $\Lambda R$ than the one used in the plot. However, in order to go above $R^{-1} \sim 1.5 \mathrm{TeV}$ would probably require some modifications to the model beyond the minimal scenario considered here [118]. 

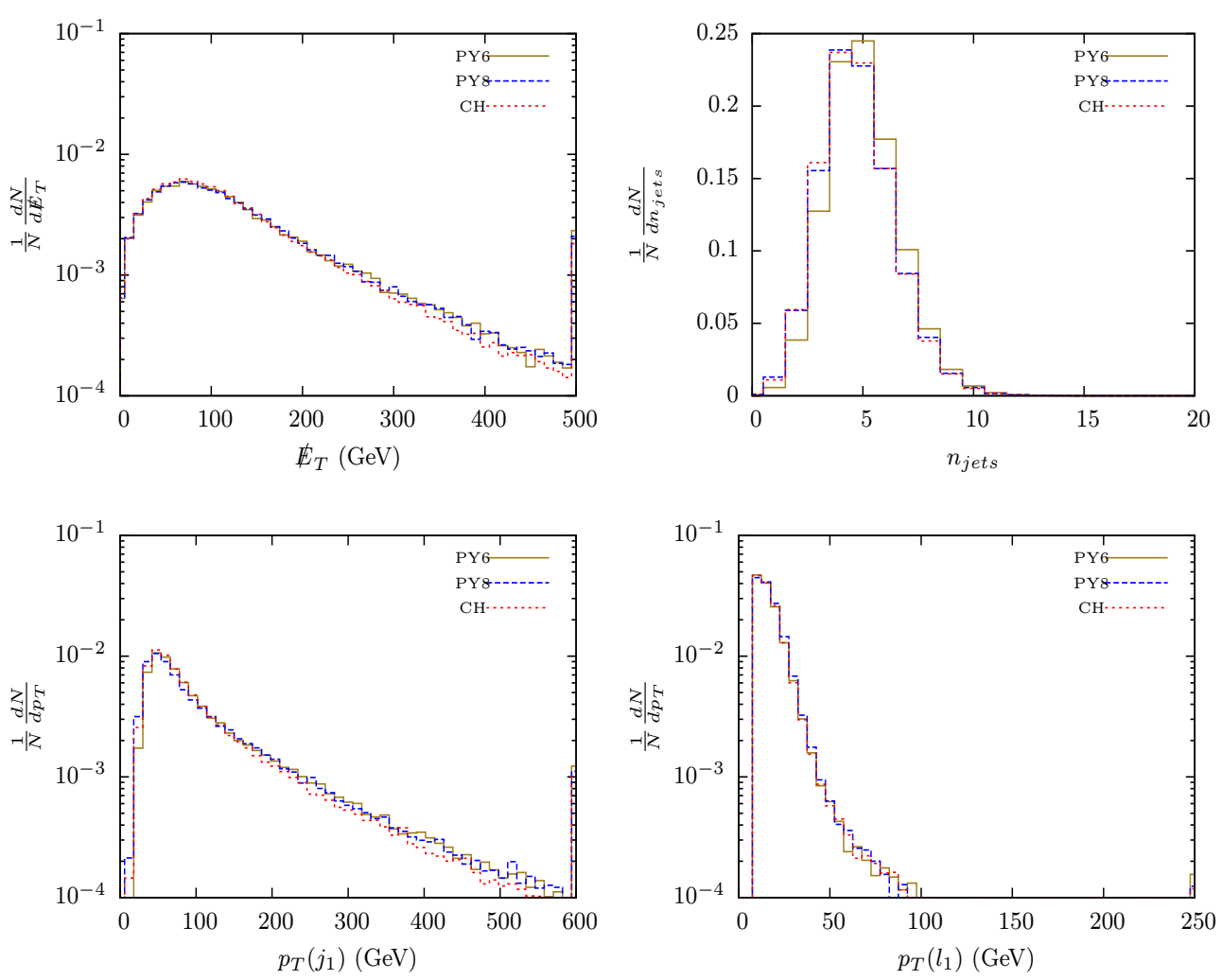

Figure 3. Some relevant kinematic distributions at the detector level for $g_{1}^{\star} g_{1}^{\star}$ production: the missing transverse energy $\mathbb{E}_{T}$ (upper left panel), the jet multiplicity $n_{\text {jet }}$ (upper right panel), the transverse momentum $p_{T}\left(j_{1}\right)$ of the leading jet (lower left) and the transverse momentum $p_{T}\left(\ell_{1}\right)$ of the leading lepton (lower right). Parton level events were produced at LHC $8 \mathrm{TeV}$ with our modification of the Pythia6 code described in Appendix B (bisque solid lines), with our Pythia8 implementation described in Appendix C (blue, long dashed lines), or with the MUED implementation in CALCHEP [57] (red, short dashed lines).

\subsection{LHC simulation details}

At first glance, collider searches for MUED may appear challenging. The small mass splittings among the level $1 \mathrm{KK}$ partners imply relatively soft decay products. As in any model with a dark matter candidate, the generic signature is missing energy, $\mathbb{E}_{T}[119]$. However, $\mathbb{E}_{T}$ is actually measured from the total transverse momentum recoil of the visible particles in the event, and if they are relatively soft, the $\mathbb{E}_{T}$ also tends to be rather small, in spite of the large amount of missing mass. This is illustrated in the upper left panels of Figs. 3 and 4, where we show the $\mathbb{E}_{T}$ distribution in $g_{1}^{\star} g_{1}^{\star}$ production and $g_{1}^{\star} q_{1}^{*}$ production, respectively. Parton-level events were generated with our MUED implementations in Pyтhia6 and Pythia8 or with CAlcHEP [57], and run through the Pythia event generator and the Delphes detector simulator [120] as part of the standard CHECKMATE simulation chain. The figures show that the $\mathbb{E}_{T}$ distributions peak below $100 \mathrm{GeV}$, thus we need to consider the rest of the event in 

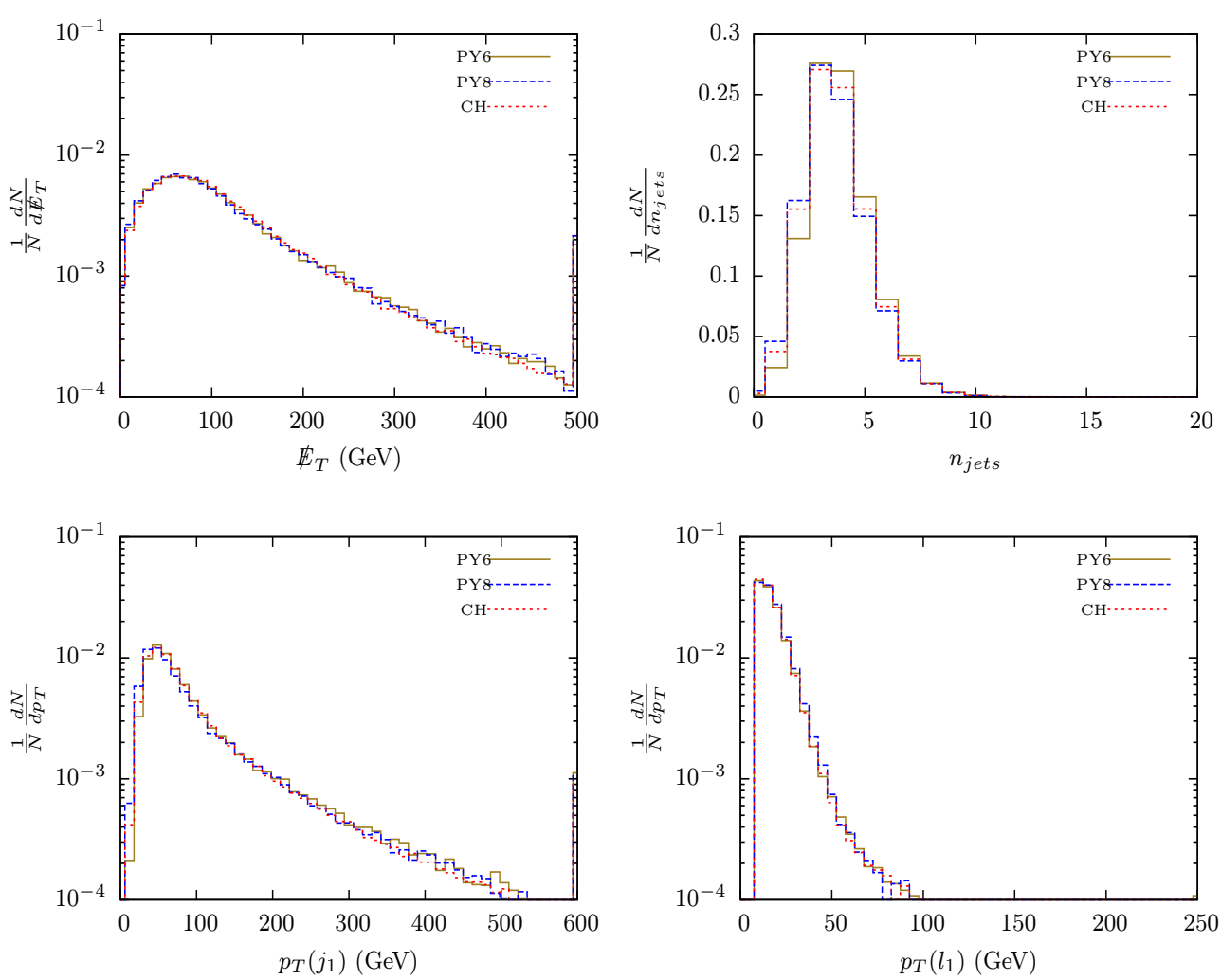

Figure 4. The same as Fig. 3, but for $g_{1}^{\star} q_{1}^{*}$ production.

order to bring the SM backgrounds under control.

The level 1 mass degeneracy also implies that the strong production cross-sections will dominate over those for electroweak production (see Fig. 6 for an illustrative example). The decays of KK quarks and KK gluons necessarily involve jets, thus we expect to have a certain amount of jets present in our signal. The upper right panels of Figs. 3 and 4 show histograms of the jet multiplicity $n_{\text {jets }}$, which depends on the production process: KK gluon events tend to have slightly more jets than KK quark events. However, those jets are not very hard, as seen in the lower left plots of Figs. 3 and 4, which depict distributions of the transverse momentum $p_{T}\left(j_{1}\right)$ of the leading jet in the event. Thus, while a simple multijet plus $\mathbb{E}_{T}$ search would have some reach [98-100], it may be beneficial to demand in addition one or more prompt leptons from the decays of the electroweak level $1 \mathrm{KK}$ bosons [78]. The lower right panels in Figs. 3 and 4 show the corresponding distributions of the transverse momentum $p_{T}\left(\ell_{1}\right)$ of the leading lepton in the event. As expected, the leptons are relatively soft - the distributions peak at the value of the lepton $p_{T}$ cut used for reconstruction. Nevertheless, there is a non-negligible tail extending to high $p_{T}$ which opens the door for a mixed strategy targeting both jets and leptons in the final state.

The main lesson from Figs. 3 and 4 is that when looking for MUED, there is no "magic" cut which would allow an easy separation of signal from background. Yet there are several 


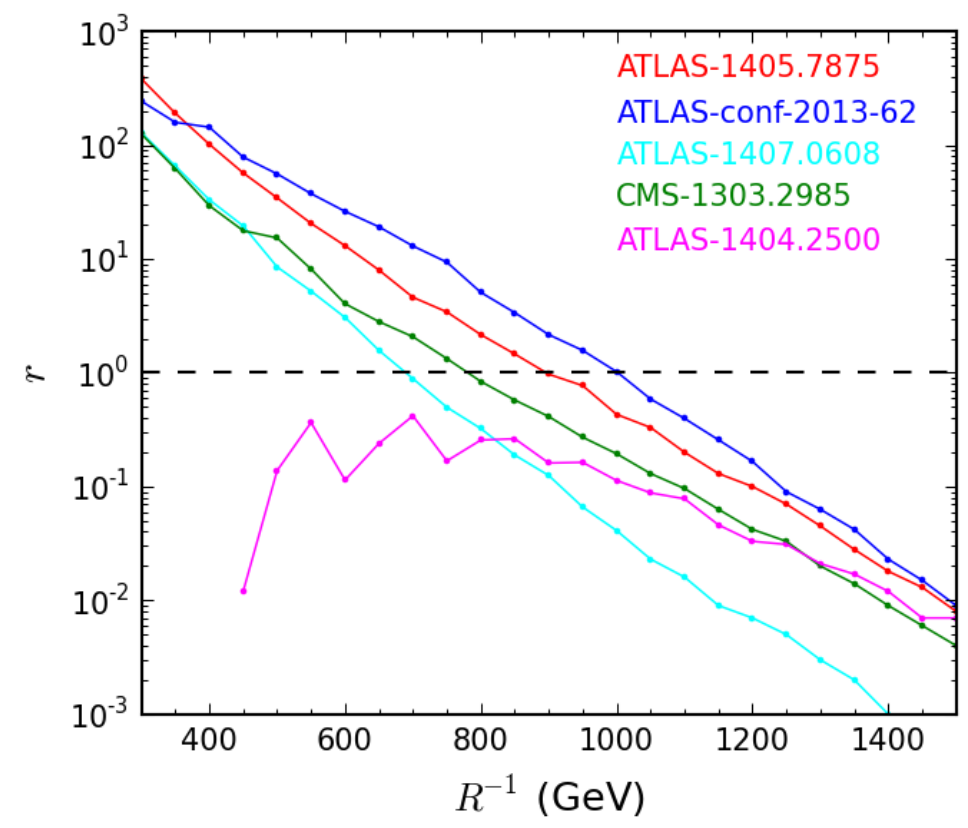

Figure 5. The $r$-value defined in eq. (4.1) as a function of $R^{-1}$ (for $\Lambda R=10$ ), for a few representative analyses from CheCKMATE.

potentially useful handles which can be utilized, and the optimal combination of jet, lepton and $\mathbb{E}_{T}$ requirements would be a function of the parameter space, i.e., $R^{-1}$.

\subsection{LHC bounds}

Checkmate [73] is one of several tools on the market which allow for an easy recasting of published LHC data. By now, a large number of LHC analyses from Run 1 have been implemented and validated in CHECKMATE [103, 104, 121-152] and we shall make use of them in deriving the limits on MUED. ${ }^{7}$ Typically, each analysis has several search regions, with different selections and cuts. For each signal region, Checkmate computes the expected number of signal events $S$ after cuts, and compares it to the $95 \%$ CL upper limit $S_{\text {exp }}^{95}$ given a signal error $\Delta S$ [77]. The model point is ruled out if the ratio

$$
r \equiv \frac{S-1.96 \Delta S}{S_{e x p}^{95}}
$$

is greater than one. In Fig. 5 we show the $r$ values as a function of $R^{-1}$ for a few of the most sensitive LHC analyses. The best limit (blue line in Fig. 5) comes from the SoftLepJ5 analysis from Ref. [103], which requires $n_{\text {jets }} \geq 5$ with $p_{T}\left(j_{1}\right)>180 \mathrm{GeV}$, one lepton with $p_{T}\left(\ell_{1}\right)<25 \mathrm{GeV}$, a minimum value for the magnitude of the missing transverse momentum $\vec{P}_{T}$ of $300 \mathrm{GeV}, m_{T}\left(\vec{p}_{T}(\ell), \vec{P}_{T}\right)>100 \mathrm{GeV}$, and $\not_{T}>0.3 m_{\text {eff }}$, where $m_{\text {eff }}$ is the scalar

\footnotetext{
${ }^{7}$ The work on incorporating Run 2 analyses in CHECKMATE is underway, but not all of them have been implemented and validated yet.
} 
sum of the $\mathbb{E}_{T}$, transverse momenta of the jets and transverse momenta of the leptons. Another sensitive analysis is the SR4jm channel [128] (red line in Fig. 5), which is an analysis with $n_{\text {jets }} \geq 4$ with $p_{T}(j)>60 \mathrm{GeV}$, zero lepton, $\mathbb{E}_{T}>160 \mathrm{GeV}, m_{\text {eff }}>1300 \mathrm{GeV}$ and $\mathbb{E}_{T}>0.4 m_{\text {eff }}$.

\section{Conclusions}

The MUED model considered here provides an interesting and motivated alternative to supersymmetry, with unique and challenging collider phenomenology. Our two main goals in this paper were:

- Validation of the existing MUED implementation in the fortran version of Pythia6 and creating a modern implementation of MUED in the $\mathrm{C}++$ version PYTHIA8.

- Demonstrating the utility of these codes when used in conjunction with a recast package like Checkmate in extracting LHC limits on the parameter space of the model.

Using the Run 1 LHC analyses incorporated in Checkmate, we have derived a lower bound on $R^{-1}$ of $1 \mathrm{TeV}$ from the SoftLepJ5 analysis from Ref. [103]. A more restrictive limit can be obtained from the $13 \mathrm{TeV}$ analyses which are currently being implemented and validated in Checkmate. Preliminary results from Ref. [153] indicate that the sensitivity will increase up to $1.4 \mathrm{TeV}$, quickly closing the window on the cosmologically motivated section of the MUED parameter space. In addition to the pair production of level $1 \mathrm{KK}$ particles, one could also use resonance searches for level $2 \mathrm{KK}$ modes to place competitive limits on the MUED parameters [44, 83-89].

Note added. While we were finishing this paper, we became aware of a concurrent study, Ref. [153], whose goal was to derive bounds on the MUED parameter space from LHC data at 8 and $13 \mathrm{TeV}$, using Herwig ++ [64] for event generation and ChEckmate for setting the limit. We thank the authors of Ref. [153] for making a preliminary draft of their paper available to us. In the overlapping regions of the two studies, the results were found to be in agreement.

\section{Acknowledgments}

This work is supported in part by a US Department of Energy grant DE-SC0010296. AD gratefully acknowledges generous financial support from the Physics Department of University of Florida towards a stay on sabbatical during which this work was undertaken. DD acknowledges support from the University of Florida Informatics Institute in the form of a Graduate Student Fellowship. The authors like to thank T. Flacke, J. S. Kim, K. Kong and J. D. Lykken for discussions. AD likes to thank P. de Aquino, D. Bourilkov, O. Mattelaer and A. Pukhov for helpful discussions on issues in handling several different packages used in this work. JB and DD thank N. Desai, S. Mrenna and J. Tattersall for technical help and advice. Participation of G. Sarangi during the initial phase of this work is also thankfully acknowledged. 


\section{A KK gluon and KK quark production: the mass-nondegenerate case}

In this appendix we present the full parton-level matrix elements for all pure $\mathrm{QCD}^{8}$ strong production processes with $2 \mathrm{KK}$ particles in the final state. Here we avoid the degenerate limit approximation (3.1) and retain the complete dependence on all relevant mass parameters (listed in the third column of Table 2). The results below have been checked in two different ways:

- In the degenerate limit (3.1) one recovers the corresponding expressions (3.6-3.17) given in Section 3.1.

- When the matrix elements were implemented in PyTHIA, the corresponding crosssections were found to agree with the numerical results from CALCHEP, as shown in Table 3.

Process 311: $g g \rightarrow g_{1}^{\star} g_{1}^{\star}$

$$
\begin{aligned}
\bar{\sum}|\mathcal{M}|_{g g \rightarrow g_{1}^{\star} g_{1}^{\star}}^{2}= & \frac{9 \alpha_{s}^{2}}{16\left(s t^{\prime} u^{\prime}\right)^{2}}\left(s^{2}+{t^{\prime}}^{2}+{u^{\prime}}^{2}\right) \\
& \times\left[6 m_{g_{1}^{\star}}^{4} s^{2}-6 m_{g_{1}^{\star}}^{2} s t^{\prime} u^{\prime}+3 t^{\prime 2} u^{\prime 2}+2 s^{2}\left(t^{\prime 2}+u^{\prime 2}\right)\right]
\end{aligned}
$$

where $t^{\prime}=t-m_{g_{1}^{\star}}^{2}$ and $u^{\prime}=u-m_{g_{1}^{\star}}^{2}$. The answer is symmetric with respect to $t^{\prime} \leftrightarrow u^{\prime}$.

Process 312: $q g \rightarrow q_{1}^{*} g_{1}^{\star}$

$$
\begin{aligned}
& \bar{\sum}|\mathcal{M}|_{q g \rightarrow q_{1}^{*} g_{1}^{\star}}^{2}=\frac{-\alpha_{s}^{2}}{72 s t^{\prime} u^{\prime 2}}\left(9 s^{2}+9 t^{\prime 2}-u^{\prime 2}+2\left(m_{g_{1}^{\star}}^{2}-m_{q_{1}^{*}}^{2}\right)\left\{-s+8 t^{\prime}+8\left(m_{g_{1}^{\star}}^{2}-m_{q_{1}^{*}}^{2}\right)\right\}\right) \\
& \times\left[2 s^{2}+2 t^{\prime 2}+\frac{m_{q_{1}^{*}}^{2}}{m_{g_{1}^{\star}}^{2}} u^{\prime 2}+\left(m_{g_{1}^{\star}}^{2}-m_{q_{1}^{*}}^{2}\right)\left\{m_{g_{1}^{\star}}^{2}\left(-4 s+2 t^{\prime}+\frac{2 s^{2}}{t^{\prime}}\right)+m_{q_{1}^{*}}^{2}\left(2 s+t^{\prime}+\frac{s^{2}}{t^{\prime}}\right)\right\}\right]
\end{aligned}
$$

where $t^{\prime}=t-m_{g_{1}^{\star}}^{2}$ and $u^{\prime}=u-m_{q_{1}^{*}}^{2}$.

Process 313a: $q q \rightarrow q_{1}^{*} q_{1}^{*}$

$$
\begin{aligned}
\bar{\sum}|\mathcal{M}|_{q q \rightarrow q_{1}^{*} q_{1}^{*}}^{2}= & \frac{\alpha_{s}^{2}}{54\left(t^{\prime}+m_{q_{1}^{*}}^{2}-m_{g_{1}^{\star}}^{2}\right)^{2}\left(u^{\prime}+m_{q_{1}^{*}}^{2}-m_{g_{1}^{\star}}^{2}\right)^{2}} \\
\times & {\left[t^{\prime 4}+u^{\prime 4}-s^{4}+6 s^{2}\left(t^{\prime 2}+u^{\prime 2}\right)+m_{q_{1}^{*}}^{2}\left(6 t^{\prime 3}+6 u^{\prime 3}-s t^{\prime} u^{\prime}\right)\right.} \\
& +\frac{m_{g_{1}^{\star}}^{2}-m_{q_{1}^{*}}^{2}}{m_{g_{1}^{\star}}^{4}}\left\{8 m_{g_{1}^{\star}}^{6}\left(s^{2}-2 s m_{q_{1}^{*}}^{2}\right)+8 m_{g_{1}^{\star}}^{4}\left(s^{3}-3 s^{2} m_{q_{1}^{*}}^{2}+3 s m_{q_{1}^{*}}^{4}\right)\right.
\end{aligned}
$$

\footnotetext{
${ }^{8} \mathrm{We}$ do not include diagrams mediated by electroweak gauge bosons and their KK modes. We omit diagrams mediated by level 2 or higher KK particles, e.g., $s$-channel diagrams with level two KK particles in the propagator.
} 


$$
\begin{aligned}
& -m_{g_{1}^{\star}}^{2}\left(2 t^{\prime 2} u^{\prime 2}-6\left(t^{\prime 3}+u^{\prime 3}\right)+3 s t^{\prime} u^{\prime} m_{q_{1}^{*}}^{2}+m_{q_{1}^{*}}^{4}\left(4 t^{\prime} u^{\prime}-\frac{19}{2} s^{2}\right)+7 s m_{q_{1}^{*}}^{6}\right) \\
& \left.\left.-2 t^{\prime 2} u^{\prime 2} m_{q_{1}^{*}}^{2}+s t^{\prime} u^{\prime} m_{q_{1}^{*}}^{4}+m_{q_{1}^{*}}^{6}\left(4 t^{\prime} u^{\prime}-\frac{s^{2}}{2}\right)-s m_{q_{1}^{*}}^{8}\right\}\right]
\end{aligned}
$$

where $t^{\prime}=t-m_{q_{1}^{*}}^{2}, u^{\prime}=u-m_{q_{1}^{*}}^{2}$.

Process 313b: $q q^{\prime} \rightarrow q_{1}^{*} q_{1}^{\prime *}$

$$
\bar{\sum}|\mathcal{M}|_{q q^{\prime} \rightarrow q_{1}^{*} q_{1}^{\prime *}}^{2}=\frac{\alpha_{s}^{2}}{18\left(t^{\prime}+m_{q_{1}^{*}}^{2}-m_{g_{1}^{\star}}^{2}\right)^{2}}\left[\frac{m_{q_{1}^{*}}^{4}}{m_{g_{1}^{\star}}^{4}} t^{2}+4 m_{q_{1}^{*}}^{2} s\left(\frac{m_{q_{1}^{*}}^{2}}{m_{g_{1}^{\star}}^{2}}-2\right)+4 s^{2}\right]
$$

where $t^{\prime}=t-m_{q_{1}^{*}}^{2}$.

Process 314: $g g \rightarrow q_{1}^{*} \bar{q}_{1}^{*}$.

$$
\bar{\sum}|\mathcal{M}|_{g g \rightarrow q_{1}^{*} \bar{q}_{1}^{*}}^{2}=\frac{-\alpha_{s}^{2}}{24 s^{2} t^{\prime 2} u^{\prime 2}}\left(4 s^{2}-9 t^{\prime} u^{\prime}\right)\left[4 m_{q_{1}^{*}}^{4} s^{2}-4 m_{q_{1}^{*}}^{2} s t^{\prime} u^{\prime}-u^{\prime} t^{\prime}\left(t^{\prime 2}+u^{\prime 2}\right)\right]
$$

where $t^{\prime}=t-m_{q_{1}^{*}}^{2}, u^{\prime}=u-m_{q_{1}^{*}}^{2}$.

Process 315: $q \bar{q} \rightarrow q_{1}^{*} \bar{q}_{1}^{*}$

$$
\begin{aligned}
& \bar{\sum}|\mathcal{M}|_{q \bar{q} \rightarrow q_{1}^{*} \bar{q}_{1}^{*}}^{2}=\frac{\alpha_{s}^{2}}{54 s^{2}\left(t^{\prime}+m_{q_{1}^{*}}^{2}-m_{g_{1}^{\star}}\right)^{2}}\left[12 s^{4}+16 s^{3} t^{\prime}+23 s^{2} t^{\prime 2}+36 s t^{\prime 3}+48 t^{\prime 4}\right. \\
& +12 \frac{m_{q_{1}^{*}}^{4}}{m_{g_{1}^{\star}}^{2}} s\left(s^{2}-s t^{\prime}+4 t^{\prime 2}\right)+4\left(m_{g_{1}^{\star}}^{2}-m_{q_{1}^{*}}^{2}\right)^{2}\left(5 s^{2}+12 s t^{\prime}+12 t^{\prime 2}+12 m_{q_{1}^{*}}^{2} s\right) \\
& +4\left(m_{g_{1}^{\star}}^{2}-m_{q_{1}^{*}}^{2}\right)\left(2 s^{3}-8 s^{2} t^{\prime}-22 s t^{\prime 2}-24 t^{\prime 3}+m_{g_{1}^{\star}}^{2} s^{2}+m_{q_{1}^{*}}^{2} s\left(s-24 t^{\prime}\right)\right. \\
& \left.+\frac{m_{g_{1}^{\star}}^{2}-m_{q_{1}^{*}}^{2}}{m_{g_{1}^{\star}}^{2}}\left\{s t^{\prime 2}\left(3 s-4 t^{\prime}\right)+m_{q_{1}^{*}}^{2} s t^{\prime}\left(8 s-52 t^{\prime}\right)-4 m_{q_{1}^{*}}^{4} s^{2}+3 s^{2} t^{\prime 2} \frac{m_{q_{1}^{*}}^{2}}{m_{g_{1}^{\star}}^{2}}\right\}\right]
\end{aligned}
$$

where $t^{\prime}=t-m_{q_{1}^{*}}^{2}$.

Process 316: $q \bar{q}^{\prime} \rightarrow q_{1}^{\bullet} \bar{q}_{1}^{\prime \circ}$

$$
\begin{aligned}
\bar{\sum}|\mathcal{M}|_{q \bar{q}^{\prime} \rightarrow q_{1}^{\bullet} \bar{q}_{1}^{\prime \circ}}^{2} & =\frac{\alpha_{s}^{2}}{18\left(m_{g_{1}^{\star}}^{2}-t_{D}^{\prime}-m_{q_{1}^{\bullet}}^{2}\right)\left(m_{g_{1}^{\star}}^{2}-t_{S}^{\prime}-m_{q_{1}^{\circ}}^{2}\right)} \\
& \times\left[4 s^{2}+\frac{m_{q_{1}^{\bullet}}^{2} m_{q_{1}^{\circ}}^{2}}{m_{g_{1}^{\star}}^{4}} t_{D}^{\prime} t_{S}^{\prime}-4 s m_{q_{1}^{*}}^{2}-4 s\left\{\frac{m_{q_{1}^{\circ}}^{2}}{m_{g_{1}^{\star}}^{2}}\left(m_{g_{1}^{\star}}^{2}-m_{q_{1}^{\bullet}}^{2}\right)\right\}\right]
\end{aligned}
$$

where $t_{D}^{\prime}=t-m_{q_{1}^{\bullet}}^{2}, t_{S}^{\prime}=t-m_{q_{1}^{\circ}}^{2}$. 
Process 317: $q \bar{q}^{\prime} \rightarrow q_{1}^{*} \bar{q}_{1}^{*}$

$$
\bar{\sum}|\mathcal{M}|_{q \bar{q}^{\prime} \rightarrow q_{1}^{*} \bar{q}_{1}^{\prime *}}^{2}=\frac{\alpha_{s}{ }^{2}}{18\left(m_{g_{1}^{\star}}^{2}-t^{\prime}-m_{q_{1}^{*}}^{2}\right)^{2}}\left[4 \frac{m_{q_{1}^{*}}^{4}}{m_{g_{1}^{\star}}^{2}} s+4 s^{2}+8 s t^{\prime}+\left(4+\frac{m_{q_{1}^{*}}^{4}}{m_{g_{1}^{\star}}^{4}}\right) t^{\prime 2}\right]
$$

where $t^{\prime}=t-m_{q_{1}^{*}}^{2}$.

Process 318a: $q q \rightarrow q_{1}^{\bullet} q_{1}^{\circ}$

$$
\begin{aligned}
\bar{\sum}|\mathcal{M}|_{q q \rightarrow q_{1}^{\bullet} q_{1}^{\circ}}^{2} & =\frac{\alpha_{s}^{2}}{9 t^{\prime 2} u^{\prime 2}}\left[\frac{2 m_{q_{1}^{\bullet}}^{2} m_{q_{1}^{\circ}}^{2}}{m_{g_{1}^{\star}}^{2}} s^{\prime}\left(t^{\prime 2}+u^{\prime 2}\right)+2 s^{\prime 4}-8 s^{\prime 2} t^{\prime} u^{\prime}+\left(4+\frac{m_{q_{1}^{\bullet}}^{2} m_{q_{1}^{\circ}}^{2}}{m_{g_{1}^{\star}}^{4}}\right) t^{\prime 2} u^{\prime 2}\right. \\
& +4\left(m_{q_{1}^{\circ}}^{4}-m_{q_{1}^{\bullet}}^{4}\right)\left(m_{q_{1}^{\bullet}}^{2}-m_{g_{1}^{\star}}^{2}\right)^{2}+\left(m_{q_{1}^{\bullet}}^{4}-m_{q_{1}^{\circ}}^{4}\right)\left(m_{q_{1}^{\bullet}}^{2}-m_{g_{1}^{\star}}^{2}\right)\left(2 t^{\prime}-s^{\prime}\right) \\
& +\frac{\left(t^{\prime 2}+u^{\prime 2}\right)}{2 m_{g_{1}^{\star}}^{4}}\left\{4 m_{g_{1}^{\star}}^{6}\left(m_{g_{1}^{\star}}^{2}-m_{q_{1}^{\circ}}^{2}\right)+3 m_{q_{1}^{\bullet}}^{2} m_{q_{1}^{\circ}}^{2} m_{g_{1}^{\star}}^{2}\left(m_{q_{1}^{\bullet}}^{2}-m_{g_{1}^{\star}}^{2}\right)\right. \\
& \left.+3 m_{q_{1}^{\bullet}}^{2} m_{g_{1}^{\star}}^{2}\left(m_{q_{1}^{\circ}}^{4}-m_{g_{1}^{\star}}^{4}\right)+m_{q_{1}^{\bullet}}^{2}\left(m_{q_{1}^{\bullet}}^{2} m_{q_{1}^{\circ}}^{4}-m_{g_{1}^{\star}}^{6}\right)\right\}+2\left(m_{q_{1}^{\bullet}}^{4}-m_{q_{1}^{\circ}}^{4}\right) s^{\prime} t^{\prime} \\
& +\left(2 m_{g_{1}^{\star}}^{2}-m_{q_{1}^{\bullet}}^{2}-m_{q_{1}^{\circ}}^{2}\right)\left(6-\frac{m_{q_{1}^{*}}^{2} m_{q_{1}^{\circ}}^{2}}{2 m_{g_{1}^{\star}}^{4}}\right) s^{\prime} t^{\prime} u^{\prime} \\
& \left.-2\left(2 m_{g_{1}^{\star}}^{2}-m_{q_{1}^{\circ}}^{2}-m_{q_{1}^{\bullet}}^{2}\right) s^{\prime 3}\right]
\end{aligned}
$$

where $t^{\prime}=t-m_{g_{1}^{\star}}^{2}, u^{\prime}=u-m_{g_{1}^{\star}}^{2}, s^{\prime}=s+\left(2 m_{g_{1}^{\star}}^{2}-m_{q_{1}^{\bullet}}^{2}-m_{q_{1}^{\circ}}^{2}\right)$.

Process 318b: $q q^{\prime} \rightarrow q_{1}^{\bullet} q_{1}^{\circ}$

$$
\begin{aligned}
\bar{\sum}|\mathcal{M}|_{q q^{\prime} \rightarrow q_{1}^{\bullet} q_{1}^{\prime \circ}}^{2} & =\frac{-\alpha_{s}^{2}}{18\left(m_{g_{1}^{\star}}^{2}-t_{D}^{\prime}-m_{q_{1}^{\bullet}}^{2}\right)\left(m_{g_{1}^{\star}}^{2}-t_{S}^{\prime}-m_{q_{1}^{\circ}}^{2}\right)} \\
& \times\left[4 s^{2}+4 s\left(t_{S}^{\prime}+t_{D}^{\prime}\right)+4 \frac{m_{q_{1}^{\circ}}^{2} m_{q_{1}^{\bullet}}^{2}}{m_{g_{1}^{\star}}^{2}} s+t_{D}^{\prime} t_{S}^{\prime}\left(4+\frac{m_{q_{1}^{\bullet}}^{2} m_{q_{1}^{\circ}}^{2}}{m_{g_{1}^{\star}}^{4}}\right)\right]
\end{aligned}
$$

where $t_{D}^{\prime}=t-m_{q_{1}^{\bullet}}^{2}, t_{S}^{\prime}=t-m_{q_{1}^{\circ}}^{2}$.

Process 319: $q \bar{q} \rightarrow q_{1}^{\prime *} \bar{q}_{1}^{\prime *}$

$$
\bar{\sum}|\mathcal{M}|_{q \bar{q} \rightarrow q_{1}^{\prime *} \bar{q}_{1}^{\prime *}}^{2}=\frac{4 \alpha_{s}^{2}}{9 s^{2}}\left(2 m_{q_{1}^{*}}^{2} s+t^{\prime 2}+u^{\prime 2}\right)
$$

where $t^{\prime}=t-m_{q_{1}^{*}}^{2}, u^{\prime}=u-m_{q_{1}^{*}}^{2}$.

Process 320: $q \bar{q} \rightarrow g_{1}^{\star} g_{1}^{\star}$

The corresponding expression is too long and we do not present it here. It can be found in the code. 


\section{B Implementation of MUED in PYTHIA6}

The default implementation of MUED in PYTHIA6 is improved mainly along the following lines.

- It now allows for different masses for various level $1 \mathrm{KK}$ excitations. These may appear both in the final states and in the propagators of $t / u$-channel contributions. Thus, implementing correct kinematics and ensuring proper interferences of diagrams require generalization of some of the routines handling the kinematics and incorporation of suitable squared matrix elements.

- It now contains a new subprocess in the form of $q \bar{q} \rightarrow g_{1}^{*} g_{1}^{*}$. A couple of production modes (which originally had some subprocesses clubbed together) have now been split. This action becomes necessary in view of the issue discussed in the previous item.

- For some processes, corrections in the expressions for the existing squared matrix elements have been made.

To achieve these, some modifications are carried out in several routines of the original Pythia6 implementation. These are outlined below.

- The arrays like ISET, KFPR and PROC are suitably modified to find and work with the newly added ISUB entries. These arrays are contained in the block PYDATA.

- Subroutines like PYRAND and PYSCAT are modified to set the intended initial and final states appropriately.

- The array MAPPR, defined in the subroutine PYSIGH, stores a flag to call PYXUED. This is modified to make calls to the new ISUB entries possible.

- The subroutine PYMAXI calculates the maximum cross section for a process. This is also modified to make calls to the new ISUB entries possible.

- The subroutine PYXUED is modified and extended suitably to include all possible $2 \rightarrow 2$ strong-processes leading to a pair of level $1 \mathrm{KK}$ gluon and/or KK quarks.

The new driver code 'mued.f' is copied below verbatim. It is only different from the original driver file in having the provision for some extra ISUB values as shown in Table 3.

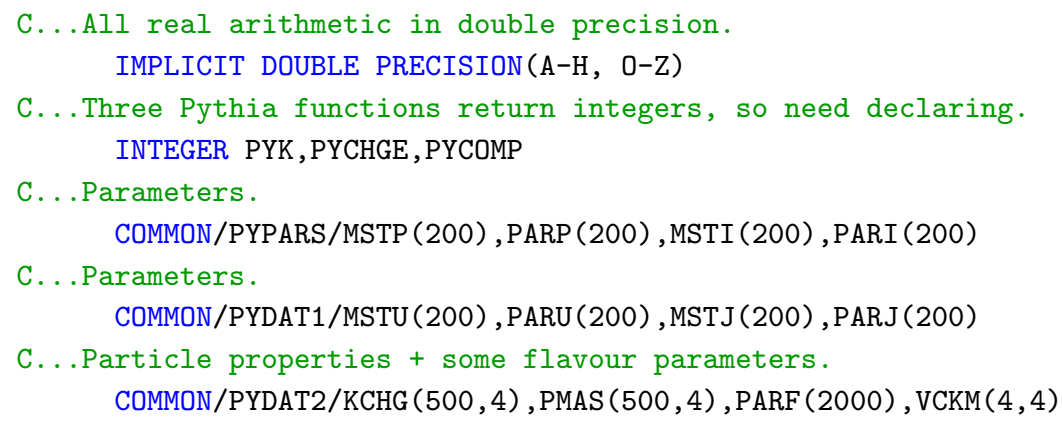




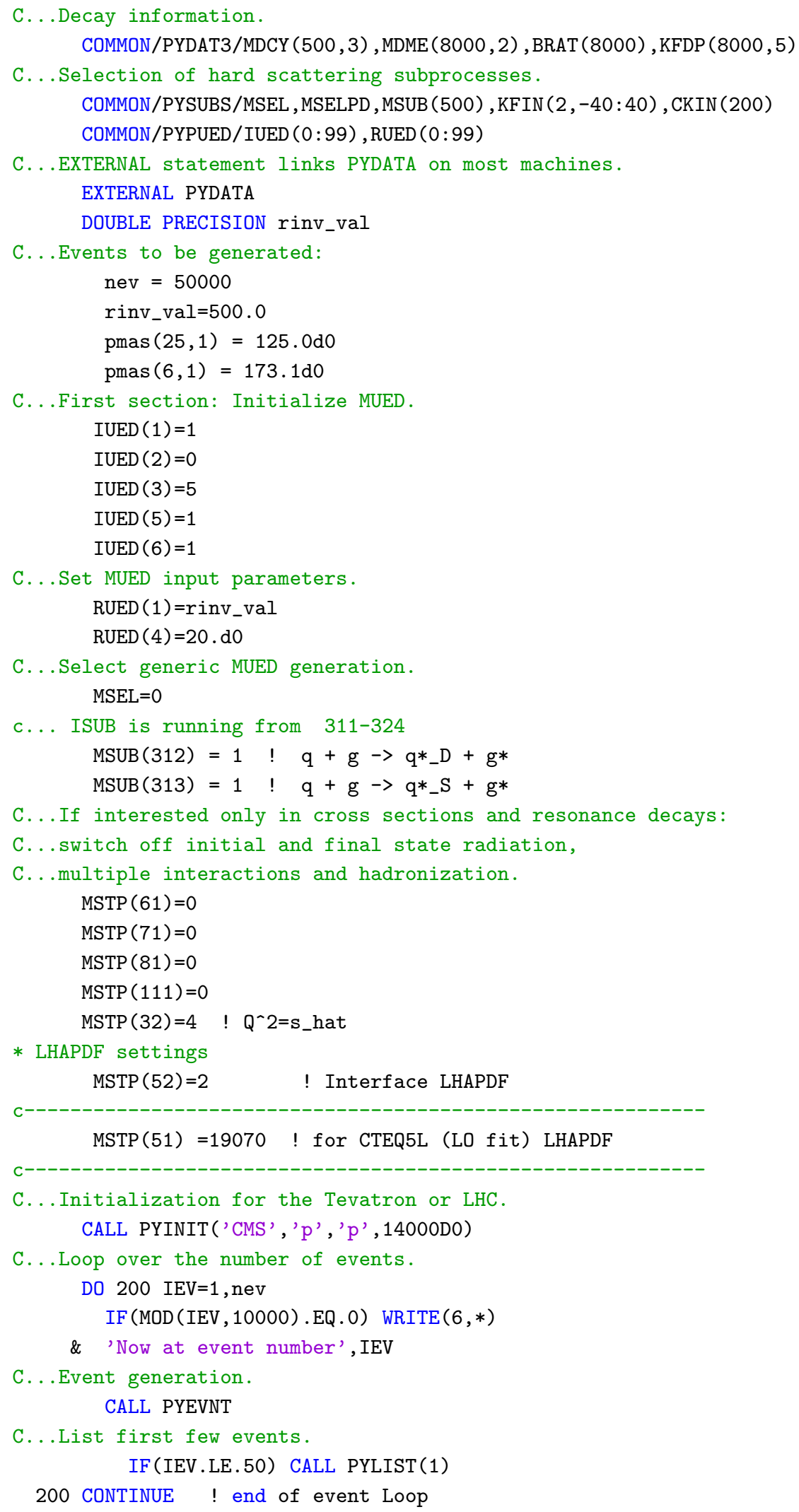




\begin{tabular}{|c|c|c|}
\hline Sub Processes (including c.c.) & PYTHIA8 identifier & PYTHIA8 class \\
\hline$g g \rightarrow g_{1}^{\star} g_{1}^{\star}$ & gg2KGKG & Sigma2gg2KGKG \\
\hline$q_{i} g \rightarrow q_{i}^{\bullet} g_{1}^{\star}, q_{i}^{\circ} g_{1}^{\star}$ & qg2KQKG & Sigma2qg2KQKG \\
\hline$q_{i} q_{j} \rightarrow q_{i}^{\bullet} q_{j}^{\bullet}, q_{i}^{\circ} q_{j}^{\circ}, q_{i}^{\bullet} q_{j}^{\circ}$ & qq2KQKQ & Sigma2qq2KQKQ \\
\hline$q_{i} \bar{q}_{j} \rightarrow q_{i}^{\bullet} \bar{q}_{j}^{\bullet}, q_{i}^{\circ} \bar{q}_{j}^{\circ}, q_{i}^{\bullet} \bar{q}_{j}^{\circ}$ & qqbar2KQKQbar & Sigma2qqbar2KQKQbar \\
\hline$q_{i} \bar{q}_{i} \rightarrow q_{j}^{\bullet} \bar{q}_{j}^{\bullet}, q_{j}^{\circ} \bar{q}_{j}^{\circ}(i \neq j)$ & qqbar2KQpKQpbar & Sigma2qqbar2KQpKQpbar \\
\hline$q \bar{q} \rightarrow g_{1}^{\star} g_{1}^{\star}$ & qqbar2KGKG & Sigma2qqbar2KGKG \\
\hline$g g \rightarrow q^{\bullet} \bar{q}^{\bullet}, q^{\circ} \bar{q}^{\circ}$ & gg2KQKQbar & Sigma2gg2KQKQbar \\
\hline$q_{i} \bar{q}_{j} \rightarrow V_{1} V_{1}$ & qqbar2W1W1 & Sigma2qqbar2W1W1 \\
\hline & qqbar2Z1Z1 & Sigma2qqbar2Z1Z1 \\
\hline & qqbar2Z1W1 & Sigma2qqbar2Z1W1 \\
\hline & qqbar2B1V1 & Sigma2qqbar2B1V1 \\
\hline
\end{tabular}

Table 4. The generic processes implemented in PYTHIA8.

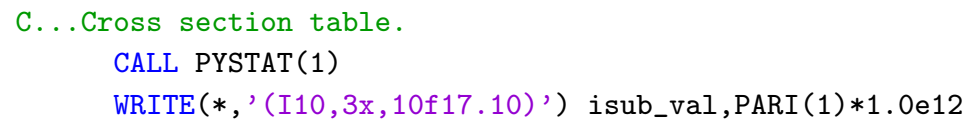

The necessary files for the improved MUED implementation in PYTHIA6 can be downloaded from http://www.hri.res.in/ jyotiranjan/download.html.

\section{Implementation of MUED in PYTHIA8}

It is easier to add new models to PythiA8 (when compared to PYTHIA6) thanks to its highly modular structure. In our implementation of the MUED scenario, we broadly adhere to the strategy followed for SUSY in PythiA8. We retain the internal routines of PYTHIA 8 and just add plugins for our purpose. Pyтнia 8 depends on other spectrum generators to find the spectrum it likes to work with. Thus, the MUED inputs and the resulting spectrum (along with other related information) are fed to PYTHIA8 from CALCHEP via SLHA format. However, one can indicate and use input masses of his/her choice directly in the code. The SLHA file also contains the information on the decays (widths and branching fractions) of all the level $1 \mathrm{KK}$ excitations. To compare the Pythia 8 results with those from CaLcHEP, we use $\alpha_{s}$ obtained directly from the LHAPDF parton distribution in use. However, $\alpha_{s}$ from PYTHIA8 itself can be used just by toggling the switch GetAlphasFromPDF(true) to GetAlphasFromPDF (false).

We have made an extensive use of ResonanceWidths class and Sigma2Process class to implement resonant 2 -body decays and $2 \rightarrow 2$ hard scattering processes, respectively. In PYTHIA8 we incorporate all the MUED processes implemented in the original version of Pythia6 plus the missing ones that we added in our Pythia6 implementation. In addition, we included pair-production processes for level $1 \mathrm{KK}$ gauge bosons. For pair-production 

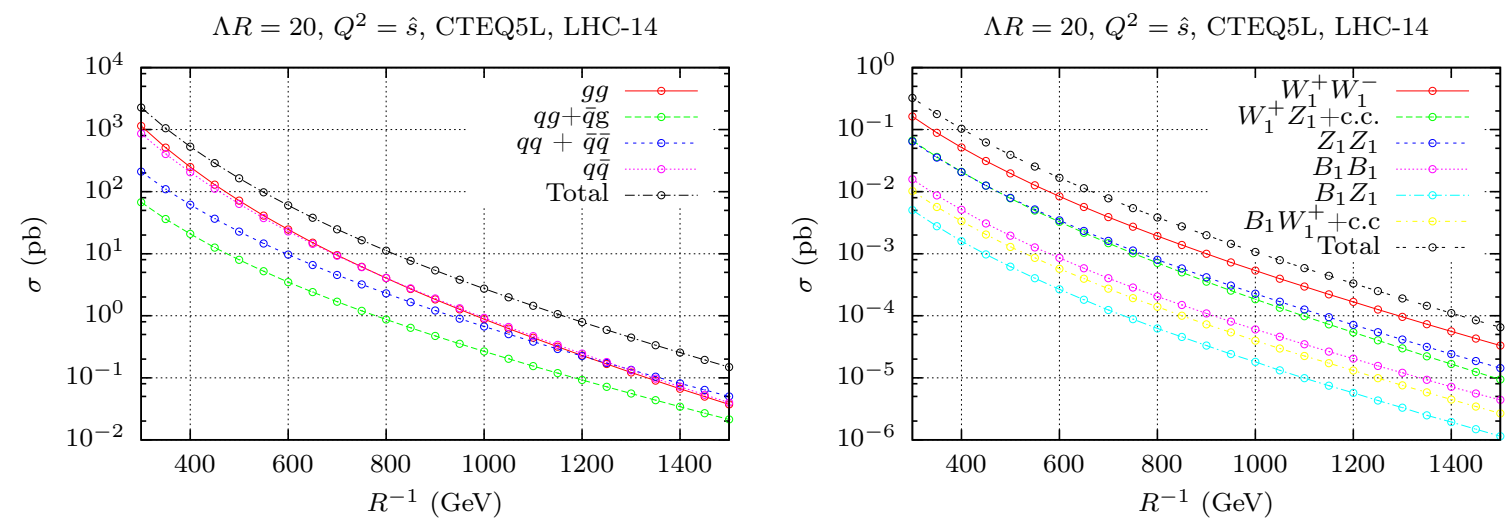

Figure 6. Left(Right): Production cross sections of strong (weak) KK particles at $14 \mathrm{TeV}$ LHC for $\Lambda R=20$ as a function of $R^{-1}$. The individual contributions are categorized by the type of initial state partons (left panel) or final state particles (right panel).

of level $1 \mathrm{KK}$ quarks, processes involving only strong interaction are considered. Processes that involve virtual level-2 gauge bosons are not included as of now, as is also the case with Pythia6. The generic $2 \rightarrow 2$ scattering processes implemented in Pythia8 are listed in Table 4 and the corresponding production cross-sections are illustrated in Fig. 6, as a function of $R^{-1}$, for $\Lambda R=20$.

The driver file 'mued.cc' for working with the MUED scenario is pasted below verbatim. The example process indicated there is $q g \rightarrow q_{1}^{\bullet} g_{1}^{\star}, q_{1}^{\circ} g_{1}^{\star}$.

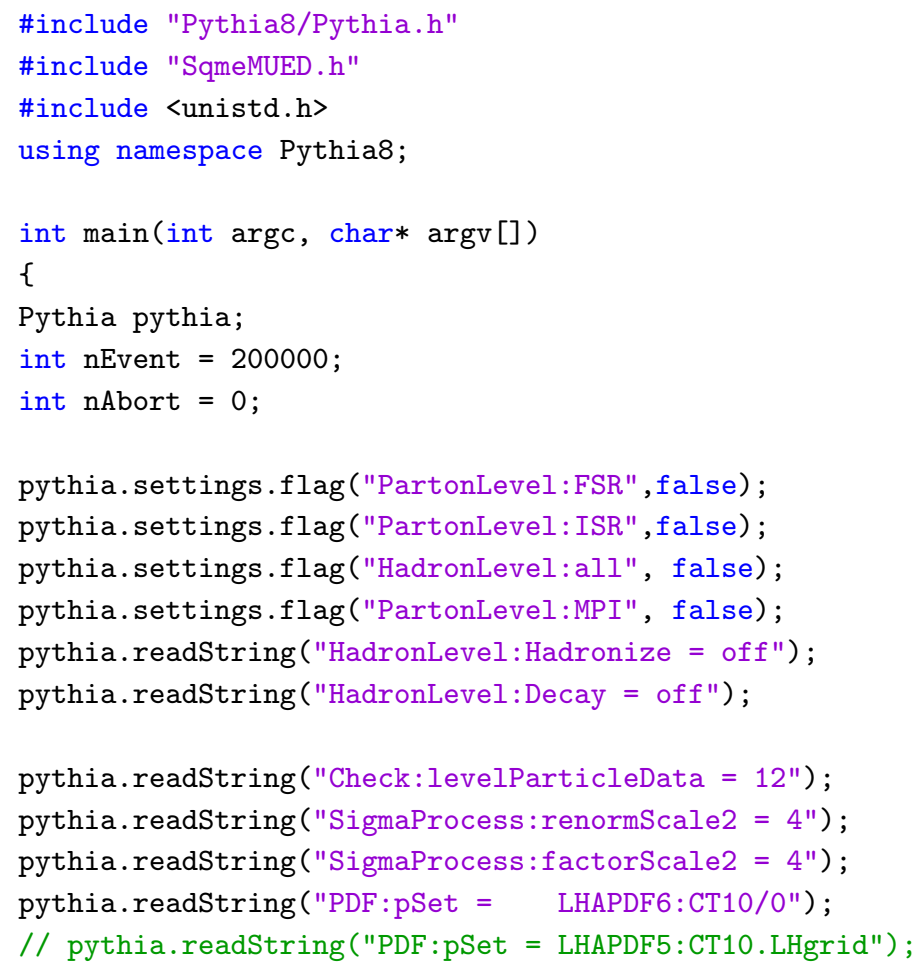




\begin{tabular}{|c|c|c|c|c|c|c|}
\hline Particles & $g_{1}$ & $B_{1}$ & $Z_{1}$ & $W_{1}^{ \pm}$ & $q_{1}^{\bullet}\left(\ell_{1}^{\bullet}\right)$ & $q_{1}^{\circ}\left(\ell_{1}^{\circ}\right)$ \\
\hline (PDG) ID & 5500021 & 5500022 & 5500023 & 5500024 & $\begin{array}{c}5500000 \\
+\operatorname{PDG}(q / \ell)\end{array}$ & $\begin{array}{c}6500000 \\
+\mathrm{PDG}(q / \ell)\end{array}$ \\
\hline
\end{tabular}

Table 5. PDG codes of the KK particles. Here PDG $(q / \ell)$ stands for the PDG code of a SM quark $q$ or a SM lepton $\ell$.

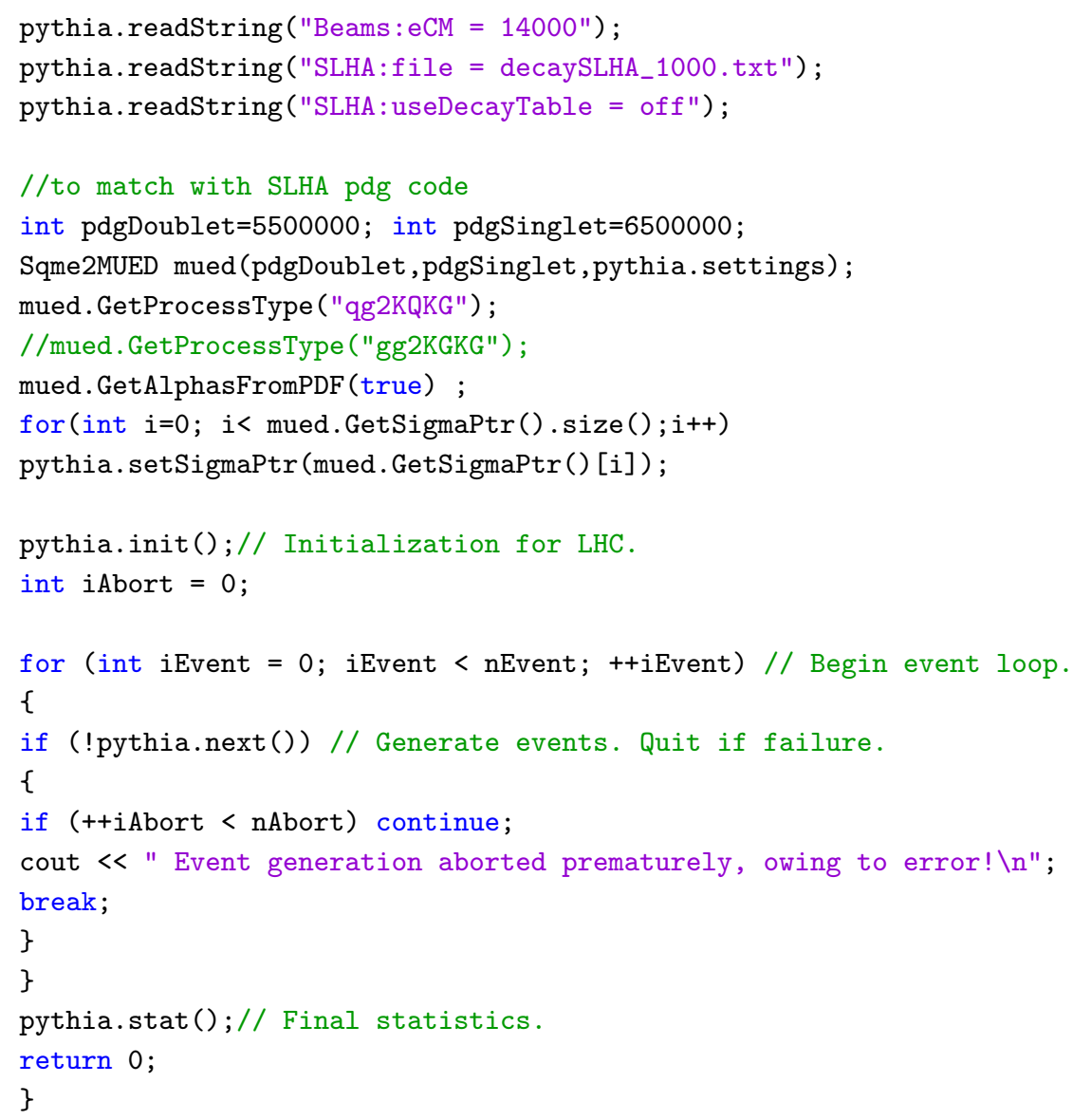

The PDG IDs assigned to various level $1 \mathrm{KK}$ excitations are shown in Table 5.

The necessary files for the MUED implementation in PYTHIA8 can be downloaded from http://www.hri.res.in/ jyotiranjan/download.html.

\section{References}

[1] S. Ask et al., "From Lagrangians to Events: Computer Tutorial at the MC4BSM-2012 Workshop," arXiv:1209.0297 [hep-ph].

[2] D. Debnath, J. S. Gainer and K. T. Matchev, "Discoveries far from the Lamppost with Matrix Elements and Ranking," Phys. Lett. B 743, 1 (2015) doi:10.1016/j.physletb.2015.02.020 [arXiv:1405.5879 [hep-ph]]. 
[3] D. Debnath, J. S. Gainer, D. Kim and K. T. Matchev, "Edge Detecting New Physics the Voronoi Way," Europhys. Lett. 114, no. 4, 41001 (2016) doi:10.1209/0295-5075/114/41001 [arXiv:1506.04141 [hep-ph]].

[4] S. P. Martin, "A Supersymmetry primer," Adv. Ser. Direct. High Energy Phys. 21, 1 (2010) [Adv. Ser. Direct. High Energy Phys. 18, 1 (1998)] doi:10.1142/9789812839657_0001, 10.1142/9789814307505_0001 [hep-ph/9709356].

[5] H. E. Haber, "Introductory low-energy supersymmetry," In *Boulder 1992, Proceedings, Recent directions in particle theory* 589-686, and Calif. Univ. Santa Cruz - SCIPP 92-033 (93/04,rec.Jun.) 98 p [hep-ph/9306207].

[6] A. Djouadi et al. [MSSM Working Group Collaboration], "The Minimal supersymmetric standard model: Group summary report," hep-ph/9901246.

[7] C. F. Berger, J. S. Gainer, J. L. Hewett and T. G. Rizzo, "Supersymmetry Without Prejudice," JHEP 0902, 023 (2009) doi:10.1088/1126-6708/2009/02/023 [arXiv:0812.0980 [hep-ph]].

[8] G. Aad et al. [ATLAS Collaboration], "Summary of the ATLAS experiments sensitivity to supersymmetry after LHC Run 1 interpreted in the phenomenological MSSM," JHEP 1510, 134 (2015) doi:10.1007/JHEP10(2015)134 [arXiv:1508.06608 [hep-ex]].

[9] V. Khachatryan et al. [CMS Collaboration], "Phenomenological MSSM interpretation of CMS searches in pp collisions at sqrt(s) = 7 and 8 TeV," JHEP 1610, 129 (2016) doi:10.1007/JHEP10(2016)129 [arXiv:1606.03577 [hep-ex]].

[10] M. Aaboud et al. [ATLAS Collaboration], "Dark matter interpretations of ATLAS searches for the electroweak production of supersymmetric particles in $\sqrt{s}=8 \mathrm{TeV}$ proton-proton collisions," JHEP 1609, 175 (2016) doi:10.1007/JHEP09(2016)175 [arXiv:1608.00872 [hep-ex]].

[11] K. Inoue, A. Kakuto, H. Komatsu and S. Takeshita, "Aspects of Grand Unified Models with Softly Broken Supersymmetry," Prog. Theor. Phys. 68, 927 (1982) Erratum: [Prog. Theor. Phys. 70, 330 (1983)]. doi:10.1143/PTP.68.927

[12] A. H. Chamseddine, R. L. Arnowitt and P. Nath, "Locally Supersymmetric Grand Unification," Phys. Rev. Lett. 49, 970 (1982). doi:10.1103/PhysRevLett.49.970

[13] R. Barbieri, S. Ferrara and C. A. Savoy, "Gauge Models with Spontaneously Broken Local Supersymmetry," Phys. Lett. 119B, 343 (1982). doi:10.1016/0370-2693(82)90685-2

[14] L. J. Hall, J. D. Lykken and S. Weinberg, "Supergravity as the Messenger of Supersymmetry Breaking," Phys. Rev. D 27, 2359 (1983). doi:10.1103/PhysRevD.27.2359

[15] H. Baer, F. E. Paige, S. D. Protopopescu and X. Tata, "ISAJET 7.48: A Monte Carlo event generator for $p p, \bar{p} p$, and $e^{+} e^{-}$reactions," hep-ph/0001086.

[16] B. C. Allanach, "SOFTSUSY: a program for calculating supersymmetric spectra," Comput. Phys. Commun. 143, 305 (2002) doi:10.1016/S0010-4655(01)00460-X [hep-ph/0104145].

[17] A. Djouadi, J. L. Kneur and G. Moultaka, "SuSpect: A Fortran code for the supersymmetric and Higgs particle spectrum in the MSSM," Comput. Phys. Commun. 176, 426 (2007) doi:10.1016/j.cpc.2006.11.009 [hep-ph/0211331].

[18] W. Porod, "SPheno, a program for calculating supersymmetric spectra, SUSY particle decays and SUSY particle production at $e^{+} e^{-}$colliders," Comput. Phys. Commun. 153, 275 (2003) doi:10.1016/S0010-4655(03)00222-4 [hep-ph/0301101]. 
[19] B. C. Allanach, S. Kraml and W. Porod, "Theoretical uncertainties in sparticle mass predictions from computational tools," JHEP 0303, 016 (2003)

doi:10.1088/1126-6708/2003/03/016 [hep-ph/0302102].

[20] G. Belanger, S. Kraml and A. Pukhov, "Comparison of SUSY spectrum calculations and impact on the relic density constraints from WMAP," Phys. Rev. D 72, 015003 (2005) doi:10.1103/PhysRevD.72.015003 [hep-ph/0502079].

[21] D. Alves et al. [LHC New Physics Working Group Collaboration], "Simplified Models for LHC New Physics Searches," J. Phys. G 39, 105005 (2012) doi:10.1088/0954-3899/39/10/105005 [arXiv:1105.2838 [hep-ph]].

[22] [ATLAS Collaboration], "Interpretation of same-sign dilepton events at ATLAS with a simplified SUSY model," ATLAS-CONF-2011-091.

[23] H. Okawa [ATLAS Collaboration], "Interpretations of SUSY Searches in ATLAS with Simplified Models," arXiv:1110.0282 [hep-ex].

[24] S. Chatrchyan et al. [CMS Collaboration], "Interpretation of Searches for Supersymmetry with simplified Models," Phys. Rev. D 88, no. 5, 052017 (2013) doi:10.1103/PhysRevD.88.052017 [arXiv:1301.2175 [hep-ex]].

[25] CMS Collaboration [CMS Collaboration], "Further SUSY Simplified Model interpretations for Moriond 2016," CMS-PAS-SUS-16-004.

[26] C. Gutschow and Z. Marshall, "Setting limits on supersymmetry using simplified models," Journal of Visualized Experiments 81, 50419 (2013) doi:10.3791/50419 [arXiv:1202.2662 [hep-ex]].

[27] J. Barnard and B. Farmer, "A simple technique for combining simplified models and its application to direct stop production," JHEP 1406, 132 (2014) doi:10.1007/JHEP06(2014)132 [arXiv:1402.3298 [hep-ph]].

[28] G. F. Giudice and R. Rattazzi, "Theories with gauge mediated supersymmetry breaking," Phys. Rept. 322, 419 (1999) doi:10.1016/S0370-1573(99)00042-3 [hep-ph/9801271].

[29] L. Randall and R. Sundrum, "Out of this world supersymmetry breaking," Nucl. Phys. B 557, 79 (1999) doi:10.1016/S0550-3213(99)00359-4 [hep-th/9810155].

[30] G. F. Giudice, M. A. Luty, H. Murayama and R. Rattazzi, "Gaugino mass without singlets," JHEP 9812, 027 (1998) doi:10.1088/1126-6708/1998/12/027 [hep-ph/9810442].

[31] P. Z. Skands et al., "SUSY Les Houches accord: Interfacing SUSY spectrum calculators, decay packages, and event generators," JHEP 0407, 036 (2004) doi:10.1088/1126-6708/2004/07/036 [hep-ph/0311123].

[32] B. C. Allanach et al., "SUSY Les Houches Accord 2," Comput. Phys. Commun. 180, 8 (2009) doi:10.1016/j.cpc.2008.08.004 [arXiv:0801.0045 [hep-ph]].

[33] J. Alwall et al., "A Les Houches Interface for BSM Generators," doi:10.2172/921331 arXiv:0712.3311 [hep-ph].

[34] C. Degrande, C. Duhr, B. Fuks, D. Grellscheid, O. Mattelaer and T. Reiter, "UFO - The Universal FeynRules Output," Comput. Phys. Commun. 183, 1201 (2012) doi:10.1016/j.cpc.2012.01.022 [arXiv:1108.2040 [hep-ph]]. 
[35] G. Brooijmans et al., "Les Houches 2011: Physics at TeV Colliders New Physics Working Group Report," arXiv:1203.1488 [hep-ph].

[36] T. Appelquist, H. C. Cheng and B. A. Dobrescu, "Bounds on universal extra dimensions," Phys. Rev. D 64, 035002 (2001) doi:10.1103/PhysRevD.64.035002 [hep-ph/0012100].

[37] H. C. Cheng, K. T. Matchev and M. Schmaltz, "Radiative corrections to Kaluza-Klein masses," Phys. Rev. D 66, 036005 (2002) doi:10.1103/PhysRevD.66.036005 [hep-ph/0204342].

[38] H. C. Cheng and I. Low, "TeV symmetry and the little hierarchy problem," JHEP 0309, 051 (2003) doi:10.1088/1126-6708/2003/09/051 [hep-ph/0308199].

[39] C. Athanasiou, C. G. Lester, J. M. Smillie and B. R. Webber, "Distinguishing Spins in Decay Chains at the Large Hadron Collider," JHEP 0608, 055 (2006) doi:10.1088/1126-6708/2006/08/055 [hep-ph/0605286].

[40] L. T. Wang and I. Yavin, "Spin measurements in cascade decays at the LHC," JHEP 0704, 032 (2007) doi:10.1088/1126-6708/2007/04/032 [hep-ph/0605296].

[41] M. Burns, K. Kong, K. T. Matchev and M. Park, "A General Method for Model-Independent Measurements of Particle Spins, Couplings and Mixing Angles in Cascade Decays with Missing Energy at Hadron Colliders," JHEP 0810, 081 (2008) doi:10.1088/1126-6708/2008/10/081 [arXiv:0808.2472 [hep-ph]].

[42] M. Battaglia, A. Datta, A. De Roeck, K. Kong and K. T. Matchev, "Contrasting supersymmetry and universal extra dimensions at the clic multi-TeV $e^{+} e^{-}$collider," JHEP 0507, 033 (2005) [hep-ph/0502041].

[43] J. M. Smillie and B. R. Webber, "Distinguishing spins in supersymmetric and universal extra dimension models at the large hadron collider," JHEP 0510, 069 (2005) doi:10.1088/1126-6708/2005/10/069 [hep-ph/0507170].

[44] A. Datta, K. Kong and K. T. Matchev, "Discrimination of supersymmetry and universal extra dimensions at hadron colliders," Phys. Rev. D 72, 096006 (2005) Erratum: [Phys. Rev. D 72, 119901 (2005)] doi:10.1103/PhysRevD.72.096006, 10.1103/PhysRevD.72.119901 [hep-ph/0509246].

[45] A. Datta, G. L. Kane and M. Toharia, "Is it SUSY?," hep-ph/0510204.

[46] S. P. Martin, "Compressed supersymmetry and natural neutralino dark matter from top squark-mediated annihilation to top quarks," Phys. Rev. D 75, 115005 (2007) doi:10.1103/PhysRevD.75.115005 [hep-ph/0703097 [HEP-PH]].

[47] H. Baer, A. Box, E. K. Park and X. Tata, "Implications of compressed supersymmetry for collider and dark matter searches," JHEP 0708, 060 (2007) doi:10.1088/1126-6708/2007/08/060 [arXiv:0707.0618 [hep-ph]].

[48] P. Schwaller and J. Zurita, "Compressed electroweakino spectra at the LHC," JHEP 1403, 060 (2014) doi:10.1007/JHEP03(2014)060 [arXiv:1312.7350 [hep-ph]].

[49] Z. Han, G. D. Kribs, A. Martin and A. Menon, "Hunting quasidegenerate Higgsinos," Phys. Rev. D 89, no. 7, 075007 (2014) doi:10.1103/PhysRevD.89.075007 [arXiv:1401.1235 [hep-ph]].

[50] Z. Han and Y. Liu, "MT2 to the Rescue - Searching for Sleptons in Compressed Spectra at the LHC," Phys. Rev. D 92, no. 1, 015010 (2015) doi:10.1103/PhysRevD.92.015010 [arXiv:1412.0618 [hep-ph]]. 
[51] H. An, J. Gu and L. T. Wang, "Exploring the nearly degenerate stop region with sbottom decays," doi:10.3204/PUBDB-2016-05828 arXiv:1611.09868 [hep-ph].

[52] P. Konar, T. Mondal and A. K. Swain, "Demystifying compressed top squark region with kinematic variables," arXiv:1612.03269 [hep-ph].

[53] J. Fan, M. Reece and J. T. Ruderman, "Stealth Supersymmetry," JHEP 1111, 012 (2011) doi:10.1007/JHEP11(2011)012 [arXiv:1105.5135 [hep-ph]].

[54] D. S. M. Alves, M. R. Buckley, P. J. Fox, J. D. Lykken and C. T. Yu, "Stops and $\mathbb{E}_{T}$ : The shape of things to come," Phys. Rev. D 87, no. 3, 035016 (2013) doi:10.1103/PhysRevD.87.035016 [arXiv:1205.5805 [hep-ph]].

[55] S. Macaluso, M. Park, D. Shih and B. Tweedie, "Revealing Compressed Stops Using High-Momentum Recoils," JHEP 1603, 151 (2016) doi:10.1007/JHEP03(2016)151 [arXiv:1506.07885 [hep-ph]].

[56] H. C. Cheng, L. Li and Q. Qin, "Second Stop and Sbottom Searches with a Stealth Stop," JHEP 1611, 181 (2016) doi:10.1007/JHEP11(2016)181 [arXiv:1607.06547 [hep-ph]].

[57] A. Datta, K. Kong and K. T. Matchev, "Minimal Universal Extra Dimensions in CalcHEP/CompHEP," New J. Phys. 12, 075017 (2010) [arXiv:1002.4624 [hep-ph]].

[58] A. Pukhov, "CalcHEP 2.3: MSSM, structure functions, event generation, batchs, and generation of matrix elements for other packages," arXiv:hep-ph/0412191.

[59] A. Pukhov et al., "CompHEP: A Package for evaluation of Feynman diagrams and integration over multiparticle phase space," hep-ph/9908288.

[60] J. Alwall, M. Herquet, F. Maltoni, O. Mattelaer and T. Stelzer, "MadGraph 5 : Going Beyond," JHEP 1106, 128 (2011) [arXiv:1106.0522 [hep-ph]].

[61] W. Kilian, T. Ohl and J. Reuter, "WHIZARD: Simulating Multi-Particle Processes at LHC and ILC," Eur. Phys. J. C 71, 1742 (2011) doi:10.1140/epjc/s10052-011-1742-y [arXiv:0708.4233 [hep-ph]].

[62] N. D. Christensen, C. Duhr, B. Fuks, J. Reuter and C. Speckner, "Introducing an interface between WHIZARD and FeynRules," Eur. Phys. J. C 72, 1990 (2012) doi:10.1140/epjc/s10052-012-1990-5 [arXiv:1010.3251 [hep-ph]].

[63] A. Alloul, N. D. Christensen, C. Degrande, C. Duhr and B. Fuks, "FeynRules 2.0 - A complete toolbox for tree-level phenomenology," Comput. Phys. Commun. 185, 2250 (2014) doi:10.1016/j.cpc.2014.04.012 [arXiv:1310.1921 [hep-ph]].

[64] M. Bahr et al., "Herwig++ 2.1 Release Note," arXiv:0711.3137 [hep-ph].

[65] S. Hche, S. Kuttimalai, S. Schumann and F. Siegert, "Beyond Standard Model calculations with Sherpa," Eur. Phys. J. C 75, no. 3, 135 (2015) doi:10.1140/epjc/s10052-015-3338-4 [arXiv:1412.6478 [hep-ph]].

[66] P. H. Beauchemin and G. Azuelos, "Dijets and Missing Energy in ATLAS from a Universal Extra Dimension," ATL-PHYS-PUB-2005-003, ATL-COM-PHYS-2005-003.

[67] M. ElKacimi, D. Goujdami, and H. Przysiezniak, "Pythia UED", in B. C. Allanach et al., "Les Houches physics at TeV colliders 2005 beyond the standard model working group: Summary report," hep-ph/0602198. 
[68] M. ElKacimi, D. Goujdami, H. Przysiezniak and P. Z. Skands, "One Universal Extra Dimension in Pythia," Comput. Phys. Commun. 181, 122 (2010) [arXiv:0901.4087 [hep-ph]].

[69] T. Sjostrand, S. Mrenna and P. Z. Skands, "PYTHIA 6.4 Physics and Manual," JHEP 0605, 026 (2006) doi:10.1088/1126-6708/2006/05/026 [hep-ph/0603175].

[70] T. Sjostrand, S. Mrenna and P. Z. Skands, "A Brief Introduction to PYTHIA 8.1," Comput. Phys. Commun. 178, 852 (2008) doi:10.1016/j.cpc.2008.01.036 [arXiv:0710.3820 [hep-ph]].

[71] C. Macesanu, C. D. McMullen and S. Nandi, "Collider implications of universal extra dimensions," Phys. Rev. D 66, 015009 (2002) doi:10.1103/PhysRevD.66.015009 [hep-ph/0201300].

[72] See the arxiv version 2 of [71] from June 212006.

[73] M. Drees, H. Dreiner, D. Schmeier, J. Tattersall and J. S. Kim, "CheckMATE: Confronting your Favourite New Physics Model with LHC Data," Comput. Phys. Commun. 187, 227 (2015) doi:10.1016/j.cpc.2014.10.018 [arXiv:1312.2591 [hep-ph]].

[74] S. Kraml, S. Kulkarni, U. Laa, A. Lessa, W. Magerl, D. Proschofsky-Spindler and W. Waltenberger, "SModelS: a tool for interpreting simplified-model results from the LHC and its application to supersymmetry," Eur. Phys. J. C 74, 2868 (2014) doi:10.1140/epjc/s10052-014-2868-5 [arXiv:1312.4175 [hep-ph]].

[75] M. Papucci, K. Sakurai, A. Weiler and L. Zeune, "Fastlim: a fast LHC limit calculator," Eur. Phys. J. C 74, no. 11, 3163 (2014) doi:10.1140/epjc/s10052-014-3163-1 [arXiv:1402.0492 [hep-ph]].

[76] E. Conte, B. Dumont, B. Fuks and C. Wymant, "Designing and recasting LHC analyses with MadAnalysis 5," Eur. Phys. J. C 74, no. 10, 3103 (2014) doi:10.1140/epjc/s10052-014-3103-0 [arXiv:1405.3982 [hep-ph]].

[77] D. Dercks, N. Desai, J. S. Kim, K. Rolbiecki, J. Tattersall and T. Weber, "CheckMATE 2: From the model to the limit," arXiv:1611.09856 [hep-ph].

[78] H. C. Cheng, K. T. Matchev and M. Schmaltz, "Bosonic supersymmetry? Getting fooled at the CERN LHC," Phys. Rev. D 66, 056006 (2002) doi:10.1103/PhysRevD.66.056006 [hep-ph/0205314].

[79] C. Macesanu, "The Phenomenology of universal extra dimensions at hadron colliders," Int. J. Mod. Phys. A 21, 2259 (2006) doi:10.1142/S0217751X06030886 [hep-ph/0510418].

[80] G. Servant, "Status Report on Universal Extra Dimensions After LHC8," Mod. Phys. Lett. A 30, no. 15, 1540011 (2015) doi:10.1142/S0217732315400118 [arXiv:1401.4176 [hep-ph]].

[81] T. G. Rizzo, "Probes of universal extra dimensions at colliders," Phys. Rev. D 64, 095010 (2001) doi:10.1103/PhysRevD.64.095010 [hep-ph/0106336].

[82] C. Macesanu, C. D. McMullen and S. Nandi, "New signal for universal extra dimensions," Phys. Lett. B 546, 253 (2002) [arXiv:hep-ph/0207269].

[83] S. Chang, K. Y. Lee and J. Song, "The Second Kaluza-Klein neutral Higgs bosons in the minimal Universal Extra Dimension model," Phys. Lett. B 708, 144 (2012) doi:10.1016/j.physletb.2012.01.047 [arXiv:1101.0670 [hep-ph]].

[84] D. Kim, Y. Oh and S. C. Park, " $W^{\prime}$ at the LHC with $\sqrt{s}=14$ TeV: Split universal extra 
dimension model,” J. Korean Phys. Soc. 67, 1137 (2015) doi:10.3938/jkps.67.1137

[arXiv:1109.1870 [hep-ph]].

[85] S. Chang, S. Y. Shim and J. Song, "Direct bound on the minimal Universal Extra Dimension model from the $t \bar{t}$ resonance search at the Tevatron," Phys. Rev. D 86, 117503 (2012) doi:10.1103/PhysRevD.86.117503 [arXiv:1207.6876 [hep-ph]].

[86] L. Edelhuser, T. Flacke and M. Krmer, "Constraints on models with universal extra dimensions from dilepton searches at the LHC," JHEP 1308, 091 (2013) doi:10.1007/JHEP08(2013)091 [arXiv:1302.6076 [hep-ph]].

[87] A. Datta, A. Raychaudhuri and A. Shaw, "LHC limits on KK-parity non-conservation in the strong sector of universal extra-dimension models," Phys. Lett. B 730, 42 (2014) doi:10.1016/j.physletb.2014.01.027 [arXiv:1310.2021 [hep-ph]].

[88] A. Shaw, "KK-parity non-conservation in UED confronts LHC data," Eur. Phys. J. C 75, no. 1, 33 (2015) doi:10.1140/epjc/s10052-014-3245-0 [arXiv:1405.3139 [hep-ph]].

[89] U. K. Dey and A. Raychaudhuri, "KK-number non-conserving decays: Signal of $n=2$ excitations of extra-dimensional models at the LHC," Nucl. Phys. B 893, 408 (2015) doi:10.1016/j.nuclphysb.2015.02.007 [arXiv:1410.1463 [hep-ph]].

[90] C. Lin, "A Search for Universal Extra Dimensions in the Multi-Lepton Channel from Proton-Antiproton Collisions at 1.8 TeV," FERMILAB-THESIS-2005-69, UMI-31-94684.

[91] M. Kazana, "Discovery potential for universal extra dimensions signals with four leptons in the final state," Acta Phys. Polon. B 38, 449 (2007).

[92] P. Q. Ribeiro, "Studies of the discovery potential for Minimal Universal Extra Dimensions in the four lepton final state in proton-proton collisions at the Large Hadron Collider with the CMS detector," CERN-THESIS-2009-166, CMS-TS-2010-025.

[93] G. Bhattacharyya, A. Datta, S. K. Majee and A. Raychaudhuri, "Exploring the Universal Extra Dimension at the LHC," Nucl. Phys. B 821, 48 (2009) doi:10.1016/j.nuclphysb.2009.06.010 [arXiv:0904.0937 [hep-ph]].

[94] A. Belyaev, M. Brown, J. Moreno and C. Papineau, "Discovering Minimal Universal Extra Dimensions (MUED) at the LHC," JHEP 1306, 080 (2013) doi:10.1007/JHEP06(2013)080 [arXiv:1212.4858 [hep-ph]].

[95] B. Bhattacherjee, A. Kundu, S. K. Rai and S. Raychaudhuri, "Multijet Discriminators for New Physics in Leptonic Signals at the LHC," Phys. Rev. D 81, 035021 (2010) doi:10.1103/PhysRevD.81.035021 [arXiv:0910.4082 [hep-ph]].

[96] D. Choudhury, A. Datta and K. Ghosh, "Deciphering Universal Extra Dimension from the top quark signals at the CERN LHC," JHEP 1008, 051 (2010) doi:10.1007/JHEP08(2010)051 [arXiv:0911.4064 [hep-ph]].

[97] B. Bhattacherjee and K. Ghosh, "Search for the minimal universal extra dimension model at the LHC with $\sqrt{s}=7 \mathrm{TeV}$," Phys. Rev. D 83, 034003 (2011) doi:10.1103/PhysRevD.83.034003 [arXiv:1006.3043 [hep-ph]].

[98] H. Murayama, M. M. Nojiri and K. Tobioka, "Improved discovery of a nearly degenerate model: MUED using MT2 at the LHC," Phys. Rev. D 84, 094015 (2011) doi:10.1103/PhysRevD.84.094015 [arXiv:1107.3369 [hep-ph]]. 
[99] A. Datta, A. Datta and S. Poddar, "Enriching the exploration of the mUED model with event shape variables at the CERN LHC," Phys. Lett. B 712, 219 (2012) doi:10.1016/j.physletb.2012.03.012 [arXiv:1111.2912 [hep-ph]].

[100] G. Cacciapaglia, A. Deandrea, J. Ellis, J. Marrouche and L. Panizzi, "LHC Missing-Transverse-Energy Constraints on Models with Universal Extra Dimensions," Phys. Rev. D 87, no. 7, 075006 (2013) doi:10.1103/PhysRevD.87.075006 [arXiv:1302.4750 [hep-ph]].

[101] D. Choudhury and K. Ghosh, "Bounds on Universal Extra Dimension from LHC Run I and II data," Phys. Lett. B 763, 155 (2016) doi:10.1016/j.physletb.2016.10.010 [arXiv:1606.04084 [hep-ph]].

[102] A. Chakraborty, S. Chakraborty and T. S. Roy, "Chasing New Physics in Stacks of Soft Tracks," Phys. Rev. D 94, no. 11, 111703 (2016) doi:10.1103/PhysRevD.94.111703 [arXiv:1606.07826 [hep-ph]].

[103] The ATLAS collaboration [ATLAS Collaboration], "Search for squarks and gluinos in events with isolated leptons, jets and missing transverse momentum at $\sqrt{s}=8 \mathrm{TeV}$ with the ATLAS detector," ATLAS-CONF-2013-062.

[104] G. Aad et al. [ATLAS Collaboration], "Search for squarks and gluinos in events with isolated leptons, jets and missing transverse momentum at $\sqrt{s}=8 \mathrm{TeV}$ with the ATLAS detector," JHEP 1504, 116 (2015) doi:10.1007/JHEP04(2015)116 [arXiv:1501.03555 [hep-ex]].

[105] H. Yamaguchi, "Search for Supersymmetry and Universal Extra Dimensions with Degenerate Mass Spectrum in Proton-Proton Collisions at $\sqrt{s}=7 \mathrm{TeV}$," Tokyo U., 2012, http://www.icepp.s.u-tokyo.ac.jp/papers/thesis.html.

[106] L. Morvaj, "Search for Minimal Universal Extra Dimensions in the final state involving muons, jets and missing transverse energy in $\sqrt{s}=8 \mathrm{TeV}$ pp collisions with the ATLAS detector," CERN-THESIS-2014-284.

[107] S. Arrenberg, L. Baudis, K. Kong, K. T. Matchev and J. Yoo, "Kaluza-Klein Dark Matter: Direct Detection vis-a-vis LHC," Phys. Rev. D 78, 056002 (2008) doi:10.1103/PhysRevD.78.056002 [arXiv:0805.4210 [hep-ph]].

[108] M. R. Whalley, D. Bourilkov and R. C. Group, "The Les Houches accord PDFs (LHAPDF) and LHAGLUE," hep-ph/0508110.

[109] H. C. Cheng, J. L. Feng and K. T. Matchev, "Kaluza-Klein dark matter," Phys. Rev. Lett. 89, 211301 (2002) doi:10.1103/PhysRevLett.89.211301 [hep-ph/0207125].

[110] G. Servant and T. M. P. Tait, "Is the lightest Kaluza-Klein particle a viable dark matter candidate?," Nucl. Phys. B 650, 391 (2003) doi:10.1016/S0550-3213(02)01012-X [hep-ph/0206071].

[111] F. Burnell and G. D. Kribs, "The Abundance of Kaluza-Klein dark matter with coannihilation," Phys. Rev. D 73, 015001 (2006) doi:10.1103/PhysRevD.73.015001 [hep-ph/0509118].

[112] K. Kong and K. T. Matchev, "Precise calculation of the relic density of Kaluza-Klein dark matter in universal extra dimensions," JHEP 0601, 038 (2006) [hep-ph/0509119].

[113] M. Kakizaki, S. Matsumoto, Y. Sato and M. Senami, "Significant effects of second KK particles on LKP dark matter physics," Phys. Rev. D 71, 123522 (2005) doi:10.1103/PhysRevD.71.123522 [hep-ph/0502059]. 
[114] M. Kakizaki, S. Matsumoto, Y. Sato and M. Senami, "Relic abundance of LKP dark matter in UED model including effects of second KK resonances," Nucl. Phys. B 735, 84 (2006) doi:10.1016/j.nuclphysb.2005.11.022 [hep-ph/0508283].

[115] G. Belanger, M. Kakizaki and A. Pukhov, "Dark matter in UED: The Role of the second KK level," JCAP 1102, 009 (2011) doi:10.1088/1475-7516/2011/02/009 [arXiv:1012.2577 [hep-ph]].

[116] G. Belanger, F. Boudjema and A. Pukhov, "micrOMEGAs : a code for the calculation of Dark Matter properties in generic models of particle interaction," arXiv:1402.0787 [hep-ph].

[117] P. A. R. Ade et al. [Planck Collaboration], "Planck 2015 results. XIII. Cosmological parameters," Astron. Astrophys. 594, A13 (2016) doi:10.1051/0004-6361/201525830 [arXiv:1502.01589 [astro-ph.CO]].

[118] Y. Ishigure, M. Kakizaki and A. Santa, "Thermal relic abundance of the lightest Kaluza-Klein particle in phenomenological universal extra dimension models," arXiv:1611.06760 [hep-ph].

[119] J. Hubisz, J. Lykken, M. Pierini and M. Spiropulu, "Missing energy look-alikes with $100 \mathrm{pb}^{-1}$ at the LHC," Phys. Rev. D 78, 075008 (2008) doi:10.1103/PhysRevD.78.075008 [arXiv:0805.2398 [hep-ph]].

[120] J. de Favereau et al. [DELPHES 3 Collaboration], "DELPHES 3, A modular framework for fast simulation of a generic collider experiment," JHEP 1402, 057 (2014) doi:10.1007/JHEP02(2014)057 [arXiv:1307.6346 [hep-ex]].

[121] G. Aad et al. [ATLAS Collaboration], "Search for new phenomena in final states with large jet multiplicities and missing transverse momentum at $\sqrt{s}=8 \mathrm{TeV}$ proton-proton collisions using the ATLAS experiment," JHEP 1310, 130 (2013) Erratum: [JHEP 1401, 109 (2014)] doi:10.1007/JHEP10(2013)130, 10.1007/JHEP01(2014)109 [arXiv:1308.1841 [hep-ex]].

[122] G. Aad et al. [ATLAS Collaboration], "Search for direct third-generation squark pair production in final states with missing transverse momentum and two $b$-jets in $\sqrt{s}=8 \mathrm{TeV} p p$ collisions with the ATLAS detector," JHEP 1310, 189 (2013) doi:10.1007/JHEP10(2013)189 [arXiv:1308.2631 [hep-ex]].

[123] G. Aad et al. [ATLAS Collaboration], "Search for direct production of charginos and neutralinos in events with three leptons and missing transverse momentum in $\sqrt{s}=8 \mathrm{TeV} p p$ collisions with the ATLAS detector," JHEP 1404, 169 (2014) doi:10.1007/JHEP04(2014)169 [arXiv:1402.7029 [hep-ex]].

[124] G. Aad et al. [ATLAS Collaboration], "Search for direct top-squark pair production in final states with two leptons in pp collisions at $\sqrt{s}=8 \mathrm{TeV}$ with the ATLAS detector," JHEP

1406, 124 (2014) doi:10.1007/JHEP06(2014)124 [arXiv:1403.4853 [hep-ex]].

[125] G. Aad et al. [ATLAS Collaboration], "Search for direct top squark pair production in events with a $\mathrm{Z}$ boson, b-jets and missing transverse momentum in sqrt(s) $=8 \mathrm{TeV}$ pp collisions with the ATLAS detector," Eur. Phys. J. C 74, no. 6, 2883 (2014) doi:10.1140/epjc/s10052-014-2883-6 [arXiv:1403.5222 [hep-ex]].

[126] G. Aad et al. [ATLAS Collaboration], "Search for direct production of charginos, neutralinos and sleptons in final states with two leptons and missing transverse momentum in $p p$ collisions at $\sqrt{s}=8 \mathrm{TeV}$ with the ATLAS detector," JHEP 1405, 071 (2014) doi:10.1007/JHEP05(2014)071 [arXiv:1403.5294 [hep-ex]]. 
[127] G. Aad et al. [ATLAS Collaboration], "Search for supersymmetry at $\sqrt{s}=8 \mathrm{TeV}$ in final states with jets and two same-sign leptons or three leptons with the ATLAS detector," JHEP 1406, 035 (2014) doi:10.1007/JHEP06(2014)035 [arXiv:1404.2500 [hep-ex]].

[128] G. Aad et al. [ATLAS Collaboration], "Search for squarks and gluinos with the ATLAS detector in final states with jets and missing transverse momentum using $\sqrt{s}=8 \mathrm{TeV}$ proton-proton collision data," JHEP 1409, 176 (2014) doi:10.1007/JHEP09(2014)176 [arXiv:1405.7875 [hep-ex]].

[129] G. Aad et al. [ATLAS Collaboration], "Search for top squark pair production in final states with one isolated lepton, jets, and missing transverse momentum in $\sqrt{s}=8 \mathrm{TeV} p p$ collisions with the ATLAS detector," JHEP 1411, 118 (2014) doi:10.1007/JHEP11(2014)118 [arXiv:1407.0583 [hep-ex]].

[130] G. Aad et al. [ATLAS Collaboration], "Search for pair-produced third-generation squarks decaying via charm quarks or in compressed supersymmetric scenarios in $p p$ collisions at $\sqrt{s}=8 \mathrm{TeV}$ with the ATLAS detector," Phys. Rev. D 90, no. 5, 052008 (2014) doi:10.1103/PhysRevD.90.052008 [arXiv:1407.0608 [hep-ex]].

[131] G. Aad et al. [ATLAS Collaboration], "Search for new phenomena in events with a photon and missing transverse momentum in $p p$ collisions at $\sqrt{s}=8 \mathrm{TeV}$ with the ATLAS detector," Phys. Rev. D 91, no. 1, 012008 (2015) Erratum: [Phys. Rev. D 92, no. 5, 059903 (2015)] doi:10.1103/PhysRevD.92.059903, 10.1103/PhysRevD.91.012008 [arXiv:1411.1559 [hep-ex]].

[132] G. Aad et al. [ATLAS Collaboration], "Search for new phenomena in final states with an energetic jet and large missing transverse momentum in pp collisions at $\sqrt{s}=8 \mathrm{TeV}$ with the ATLAS detector," Eur. Phys. J. C 75, no. 7, 299 (2015) Erratum: [Eur. Phys. J. C 75, no. 9, 408 (2015)] doi:10.1140/epjc/s10052-015-3517-3, 10.1140/epjc/s10052-015-3639-7 [arXiv:1502.01518 [hep-ex]].

[133] G. Aad et al. [ATLAS Collaboration], "Search for supersymmetry in events containing a same-flavour opposite-sign dilepton pair, jets, and large missing transverse momentum in $\sqrt{s}=8 \mathrm{TeV}$ pp collisions with the ATLAS detector," Eur. Phys. J. C 75, no. 7, 318 (2015) Erratum: [Eur. Phys. J. C 75, no. 10, 463 (2015)] doi:10.1140/epjc/s10052-015-3661-9, 10.1140/epjc/s10052-015-3518-2 [arXiv:1503.03290 [hep-ex]].

[134] G. Aad et al. [ATLAS Collaboration], "ATLAS Run 1 searches for direct pair production of third-generation squarks at the Large Hadron Collider," Eur. Phys. J. C 75, no. 10, 510 (2015) Erratum: [Eur. Phys. J. C 76, no. 3, 153 (2016)] doi:10.1140/epjc/s10052-015-3726-9, 10.1140/epjc/s10052-016-3935-x [arXiv:1506.08616 [hep-ex]].

[135] [ATLAS Collaboration], "Search for supersymmetry at $\sqrt{s}=8 \mathrm{TeV}$ in final states with jets, missing transverse momentum and one isolated lepton," ATLAS-CONF-2012-104.

[136] G. Aad et al. [ATLAS Collaboration], "Measurements of $W^{ \pm} Z$ production cross sections in $p p$ collisions at $\sqrt{s}=8 \mathrm{TeV}$ with the ATLAS detector and limits on anomalous gauge boson self-couplings," Phys. Rev. D 93 (2016) no.9, 092004 doi:10.1103/PhysRevD.93.092004 [arXiv:1603.02151 [hep-ex]].

[137] G. Aad et al. [ATLAS Collaboration], "Search for direct pair production of the top squark in all-hadronic final states in proton-proton collisions at $\sqrt{s}=8 \mathrm{TeV}$ with the ATLAS detector," JHEP 1409, 015 (2014) doi:10.1007/JHEP09(2014)015 [arXiv:1406.1122 [hep-ex]].

[138] [ATLAS Collaboration], "Study of the spin properties of the Higgs-like particle in the 
$H \rightarrow W W^{(*)} \rightarrow e \nu \mu \nu$ channel with $21 \mathrm{fb}^{-1}$ of $\sqrt{s}=8 \mathrm{TeV}$ data collected with the ATLAS detector.," ATLAS-CONF-2013-031.

[139] [ATLAS Collaboration], "Search for supersymmetry in events with four or more leptons in $21 \mathrm{fb}^{-1}$ of pp collisions at $\sqrt{s}=8 \mathrm{TeV}$ with the ATLAS detector," ATLAS-CONF-2013-036.

[140] G. Aad et al. [ATLAS Collaboration], "Search for strong production of supersymmetric particles in final states with missing transverse momentum and at least three $b$-jets at $\sqrt{s}=8$ $\mathrm{TeV}$ proton-proton collisions with the ATLAS detector," JHEP 1410, 024 (2014) doi:10.1007/JHEP10(2014)024 [arXiv:1407.0600 [hep-ex]].

[141] G. Aad et al. [ATLAS Collaboration], "Search for direct top squark pair production in final states with two tau leptons in pp collisions at $\sqrt{s}=8 \mathrm{TeV}$ with the ATLAS detector," Eur. Phys. J. C 76, no. 2, 81 (2016) doi:10.1140/epjc/s10052-016-3897-z [arXiv:1509.04976 [hep-ex]].

[142] G. Aad et al. [ATLAS Collaboration], "Measurement of total and differential $W^{+} W^{-}$ production cross sections in proton-proton collisions at $\sqrt{s}=8 \mathrm{TeV}$ with the ATLAS detector and limits on anomalous triple-gauge-boson couplings," JHEP 1609, 029 (2016) doi:10.1007/JHEP09(2016)029 [arXiv:1603.01702 [hep-ex]].

[143] G. Aad et al. [ATLAS Collaboration], "Measurement of Spin Correlation in Top-Antitop Quark Events and Search for Top Squark Pair Production in pp Collisions at $\sqrt{s}=8 \mathrm{TeV}$ Using the ATLAS Detector," Phys. Rev. Lett. 114, no. 14, 142001 (2015) doi:10.1103/PhysRevLett.114.142001 [arXiv:1412.4742 [hep-ex]].

[144] The ATLAS collaboration [ATLAS Collaboration], "Search for an Invisibly Decaying Higgs Boson Produced via Vector Boson Fusion in $p p$ Collisions at $\sqrt{s}=8 \mathrm{TeV}$ using the ATLAS Detector at the LHC," ATLAS-CONF-2015-004.

[145] S. Chatrchyan et al. [CMS Collaboration], "Measurement of $\mathrm{W}+\mathrm{W}$ - and ZZ production cross sections in pp collisions at sqrt(s) $=8 \mathrm{TeV}$," Phys. Lett. B 721, 190 (2013) doi:10.1016/j.physletb.2013.03.027 [arXiv:1301.4698 [hep-ex]].

[146] S. Chatrchyan et al. [CMS Collaboration], "Search for supersymmetry in hadronic final states with missing transverse energy using the variables $\alpha_{T}$ and b-quark multiplicity in pp collisions at $\sqrt{s}=8 \mathrm{TeV}$," Eur. Phys. J. C 73, no. 9, 2568 (2013) doi:10.1140/epjc/s10052-013-2568-6 [arXiv:1303.2985 [hep-ex]].

[147] V. Khachatryan et al. [CMS Collaboration], "Searches for electroweak production of charginos, neutralinos, and sleptons decaying to leptons and W, Z, and Higgs bosons in pp collisions at $8 \mathrm{TeV}$," Eur. Phys. J. C 74, no. 9, 3036 (2014) doi:10.1140/epjc/s10052-014-3036-7 [arXiv:1405.7570 [hep-ex]].

[148] V. Khachatryan et al. [CMS Collaboration], "Search for dark matter, extra dimensions, and unparticles in monojet events in proton proton collisions at $\sqrt{s}=8 \mathrm{TeV}$," Eur. Phys. J. C 75, no. 5, 235 (2015) doi:10.1140/epjc/s10052-015-3451-4 [arXiv:1408.3583 [hep-ex]].

[149] V. Khachatryan et al. [CMS Collaboration], "Search for Physics Beyond the Standard Model in Events with Two Leptons, Jets, and Missing Transverse Momentum in pp Collisions at sqrt(s) = 8 TeV," JHEP 1504, 124 (2015) doi:10.1007/JHEP04(2015)124 [arXiv:1502.06031 [hep-ex]].

[150] V. Khachatryan et al. [CMS Collaboration], "Search for the production of dark matter in association with top-quark pairs in the single-lepton final state in proton-proton collisions at 
sqrt(s) = 8 TeV," JHEP 1506, 121 (2015) doi:10.1007/JHEP06(2015)121 [arXiv:1504.03198 [hep-ex]].

[151] CMS Collaboration [CMS Collaboration], "Measurement of WZ production rate," CMS-PAS-SMP-12-006.

[152] CMS Collaboration [CMS Collaboration], "Search for physics beyond the standard model in events with two opposite-sign same-flavor leptons, jets, and missing transverse energy in pp collisions at sqrt $[\mathrm{s}]=8 \mathrm{TeV}$," CMS-PAS-SUS-12-019.

[153] Nicolas Deutschmann, Thomas Flacke and Jong Soo Kim, "Current LHC constraints on Minimal Universal Extra Dimensions," in preparation.

[154] N. Desai and P. Z. Skands, "Supersymmetry and Generic BSM Models in PYTHIA 8," Eur. Phys. J. C 72, 2238 (2012) doi:10.1140/epjc/s10052-012-2238-0 [arXiv:1109.5852 [hep-ph]]. 